\title{
X-ray Line 3.55 keV from Dark Matter, Our Glass Pearls
}

Holger Bech Nielsen *

The Niels Bohr Institute

E-mail: brechanbi.dr

Colin D. Froggatt

Glasgow University

E-mail: Colin.Froggatteglasqow.ac.uk

\section{Danijel Jurman}

Rudjer Boskovic Institute, Zagreb

E-mail: Danijel.Jurmandirb.hr

\begin{abstract}
It has previously been suggested an X-ray line of photon-energy $3.55 \mathrm{keV}$ (in the emission frame), not being understood in terms of expected materials in the astrophysical sources, might come from dark matter. We confront this idea within our own model for dark matter. Our model is remarkable by not needing new physics, but only the Standard Model supplemented by a fine tuning law, the "Multiple Point Principle", restricting the values of the coupling constants and the Higgs mass in the Standard Model. The crux of the matter is that in our model the dark matter consists of cm-sized pearls, which are bubbles of a suggested new type of vacuum (called "condensate vacuum") containing some ordinary atomic matter under a very high pressure caused by the weak-interaction-scale surface tension. The inside ordinary matter is hoped to be a glassy insulator with a homolumo gap that happens to be close to the $3.55 \mathrm{keV}$, so that excitons may decay giving such X-ray radiation. The energy needed for producing this $3.55 \mathrm{keV}$-radiation is suggested to come from the surface contraction made possible, when two such pearls - very seldomly - hit each other. A major success is that we get the intensity rather well predicted order of magnitudewise in our model, using values of the parameters of our model already set up to fit properties considered before we looked at the $3.55 \mathrm{keV}$ radiation. In addition we make some theoretical considerations on the homolumo-gap model developed by two of us and I. Andric and L. Jonke.
\end{abstract}

Corfu Summer Institute 2017 'School and Workshops on Elementary Particle Physics and Gravity' 2-28 September 2017

Corfu, Greece

\footnotetext{
* Speaker.
} 


\section{Introduction}

\subsection{Who did it?:}

The main part of the present talk is concerned with dark matter pearls, that is the model of the speaker HBN and the author CDF for what dark matter really is. Recent developments by HBN and the third author DJ, in collaboration with Ivan Andric and Larisa Jonke, on the homolumo gap idea, which is used to estimate the X-ray line energy in the dark matter pearl model, are discussed at the end of this talk. An important principle underlying our dark matter model is the "Multiple Point Principle" (MPP), according to which there are several vacua with very low energy density (three such degenerate vacua in the Standard Model). Others involved in working on MPP include Don Bennett, Larisa Laperasvili, Chitta Das and Roman Nevzorov. HBN also worked on similar ideas with Niels Brene and Ivica Picek prior to the formulation of MPP.

\subsection{Genuine introduction}

Even today it is a mystery what the dark matter, needed to make the motions of stars and of galaxies in galaxy clusters consistent with Newtonian gravity, is indeed made from.

There are very many ideas as to what the dark matter could be, the more popular proposal being WIMPS - weakly interacting massive particles. Typically the speculations are that the dark matter consists of particles with masses as heavy as light atoms. However really it is so that, once the particles only interact gravitationally and one only makes observations over very large distances of the gravitational field of the dark matter as it acts say on stars, the size of the individual particles turns out to not be observable. Thus the possibility of having, as we suggest, particles of masses of order hundred thousand ton is not at all out of question:

On the figure below one can see how we imagine our dark matter particles as being of extremely high density, so that they can be of only $\mathrm{cm}$-size in spite of having the suggested masses of 100000 ton to 500000 ton. The little "Danish mountain" on the figure illustrates that it might be of the order of a $100 \mathrm{~m}$ high hill, which only the Danes would consider a mountain. 


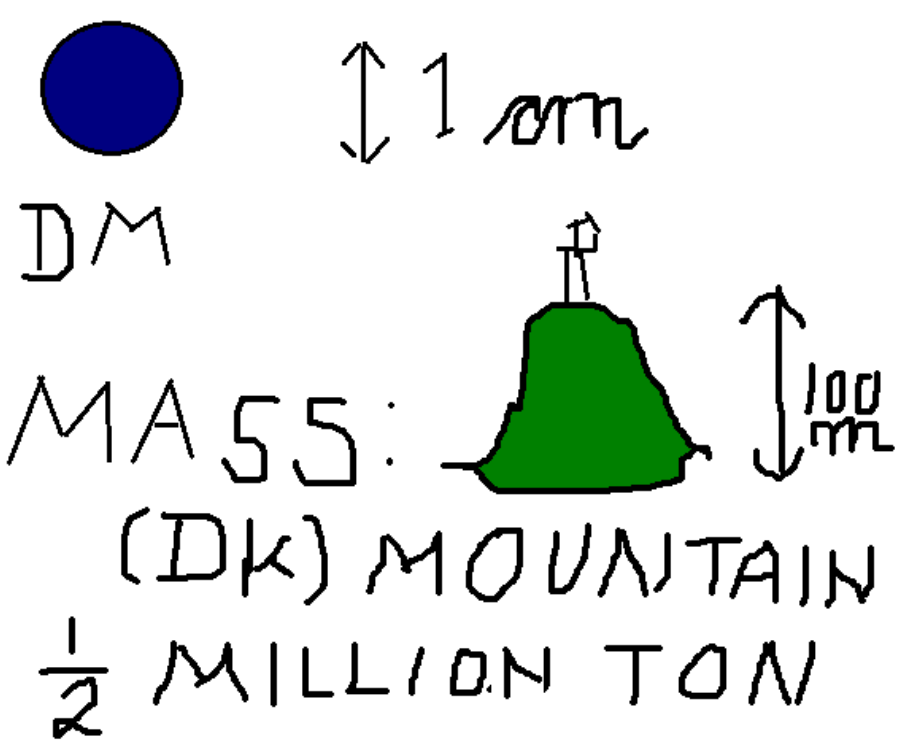

Forming a Picture of Our Dark Matter Model, Pearls:

- Our dark matter pearls [四, 口, B] are really bubbles - with the vacuum being in two different phases, the normal vacuum outside the pearl, and a "condensate vacuum" inside. Like waterdroplets have water inside and air outside. In our case the condensate consists of bound states

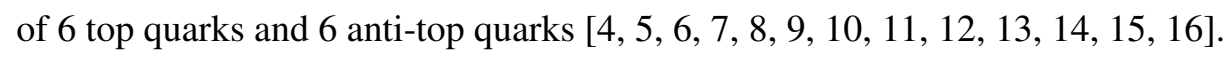

- The surface tension $S$ - being of weak interaction scale meaning $S^{1 / 3} \sim 100 \mathrm{GeV}$ - is exceedingly high from the daily life scale point of view.

$$
S=\text { "surface tension" } \simeq 10^{11} \mathrm{~kg} / \mathrm{m}^{2} \simeq 10^{28} \mathrm{~N} / \mathrm{m}
$$

An atom-broad strip of such a surface provides $10^{18} \mathrm{~N} \sim$ the weight 0 $10^{17} \mathrm{~kg}=10^{14}$ ton .

- To keep the extremely strong surface tension from squeezing/collapsing the pearl away, it must be filled with, say, ordinary matter, under extreme pressure. So there is ordinary matter inside the dark matter in our model. The density is reminiscent of that in a white dwarf say.

- If you feed a pearl with neutrons, it can take them up under release of about $10 \mathrm{MeV}$ energy per neutron. Protons would have to be shot in with big speed. But then they would also be absorbed under energy release of the order of $10 \mathrm{MeV}$.

- In the inside phase, the "condensate vacuum", we have in our model estimated that the Higgs field expectation value is about half the outside value and the quarks and the nucleons are therefore lighter inside than outside, by about $10 \mathrm{MeV}$ for nucleons. So nucleons are attracted 
towards the inside phase by a ca. $10 \mathrm{MeV}$ potential. For electrons this attraction is less (because the electron couples with a weaker Yukawa-coupling to the Higgs-field).

- Some electrons are bound electrically, a bit outside the surface with the strong tension (keeping the nucleons inside). This is imagined on the following figure as, relative to the pearl size, a thin rim of electrons around the genuine bubble.

- If you think of the rim of electrons as analogous to the swarm of electrons in an atom, you might think of our pearl as an over-dimensioned "nucleus". But the "nucleus" would not be a nucleus, but rather a clump of over-compressed ordinary matter on a background of the "condensate vacuum". In this way our pearls have the appearance of an atom with a completely over-dimensionized nucleus, that is in reality highly compressed ordinary matter on a background of the "condensate vacuum", meaning there is a lot of the bound states of 6 top +6 anti-top quarks.

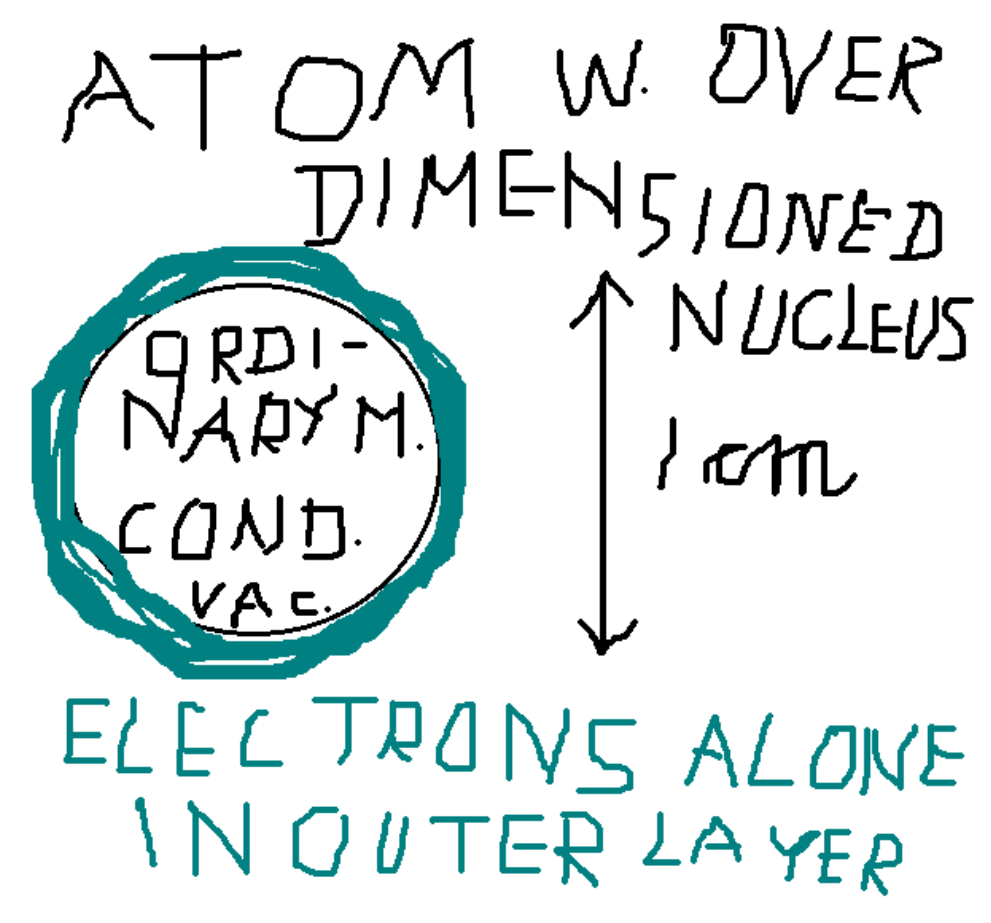

\subsection{Hope for the 3.5 keV Line in the Pearl Picture}

For the purpose of explaining the unidentified X-ray line with energy $3.5 \mathrm{keV}$ in our dark matter model, we hope that the matter inside the pearl - where the vacuum is in the "condensate vacuum" phase - is an insulator with a gap between the filled and empty electron levels being of order $3.5 \mathrm{keV}$, since then:

- After excitation and some relaxation we could have a lot of electrons in the states which should be empty (in the ground state) - especially the lowest energy ones among these -, and a lot of holes - especially also close to the fermi surface.

- This really means a lot of excitons (= pairs of hole and electron) in their low energy state. 
- Finally the excitons decay under emission of photons of the energy corresponding to the energy released by the electron falling into the hole. The gap is hoped to be $3.5 \mathrm{keV}$.

\section{Order of Magnitude of the Gap (Homolumo-gap)}

- That the gap (homolumo-gap) should be of order a few $\mathrm{keV}$ is not so unreasonable, because the ordinary matter inside the "condensate vacuum phase" in our dark matter is compressed to a density of the order $10^{14} \mathrm{~kg} / \mathrm{m}^{3}$ according to our fits. This means a compression in each of the three dimensions by a factor $\left(10^{11}\right)^{\frac{1}{3}} \sim 5000$, and we expect the homolumo gap to be crudely proportional to the inverse of the distance of neighboring atoms.

- We estimate that the homolumo-gap $\approx p_{\text {fermi }} \alpha^{2}$, which with $p_{\text {fermi }} \sim 10 \mathrm{MeV}$ can give "homolumo-gap" $1 \mathrm{keV}$.

The next figure illustrates, how we imagine the density of single electron hamiltonian eigenstates (= density of states) to vary with the eigenvalue (the energy) put along the ordinate. The important feature is that there is a range - the homolumo-gap - in which the level-density is essentially zero. It is even so that the levels below this range are filled with electrons, while those above this range (= the homolumo-gap) are empty in the ground state of the system.

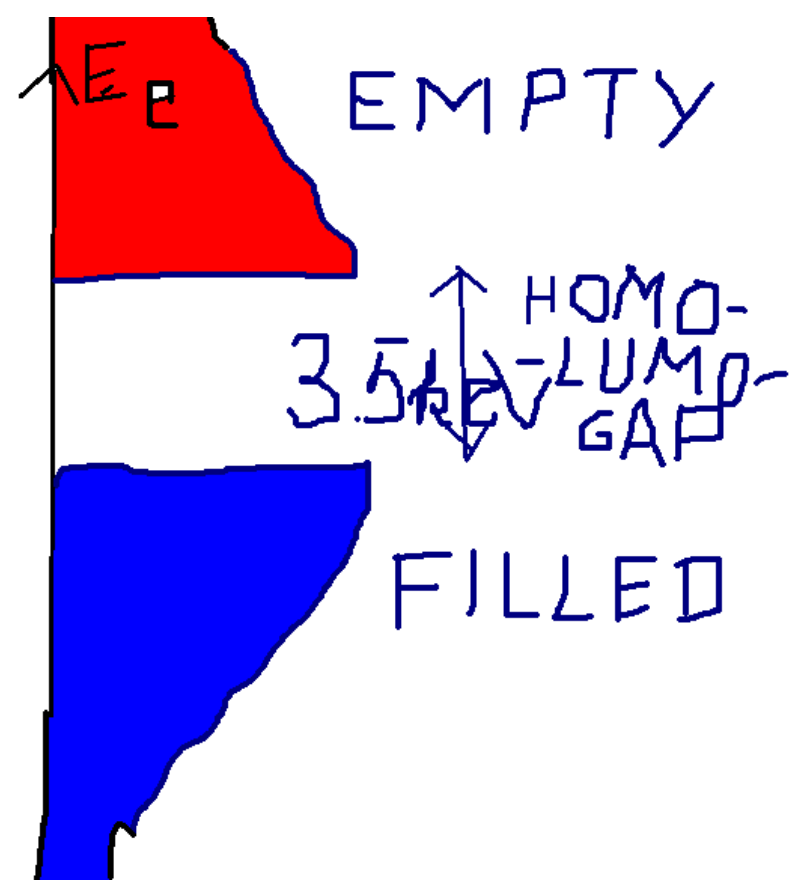

It should be stressed, that such a range with essentially no energy levels - a homolumo-gap - is expected to appear from a general mechanism of back-reaction from the electrons on the effective degrees of freedom, say the positions of the nuclei etc.. The basic idea is that the electron energy would be lowered by adjusting "whatever" (e.g. the positions of the nuclei) so as to make the filled electron levels lowered in energy, while the empty ones may stay higher. Two of us together with I. Andric and L. Jonke [ㅍ] ] have made rather general studies of this homolumo-gap effect. 


\section{The $3.5 \mathrm{keV}$ line}

The ununderstood/mysterious X-ray line was discovered by two groups, Bulbul et al.[1]8] and Boyarksy et al.[ष्प], by satellite observations (see figure below). It is so far only a tiny deviation from the X-ray spectra fitted to modeling the emission lines from expected ions. The radiation, which in the rest frame of the source has the photon energy $3.55 \mathrm{keV}$, seems to come from the centre of our galaxy as well as from galaxy clusters, places supposedly having much dark matter. An at one moment very significant source - the Perseus Cluster of Galaxies - turned out to be quite controversial, in as far as the satellite Hitomi did not see any $3.55 \mathrm{keV}$ radiation from the Perseus Cluster. This may potentially be explained by it being mixed with an absorption line. But we must admit that our own model would have difficulties with explaining absorption lines, so for our purpose it is best to hope that there is no $3.55 \mathrm{keV}$ absorption line seen.

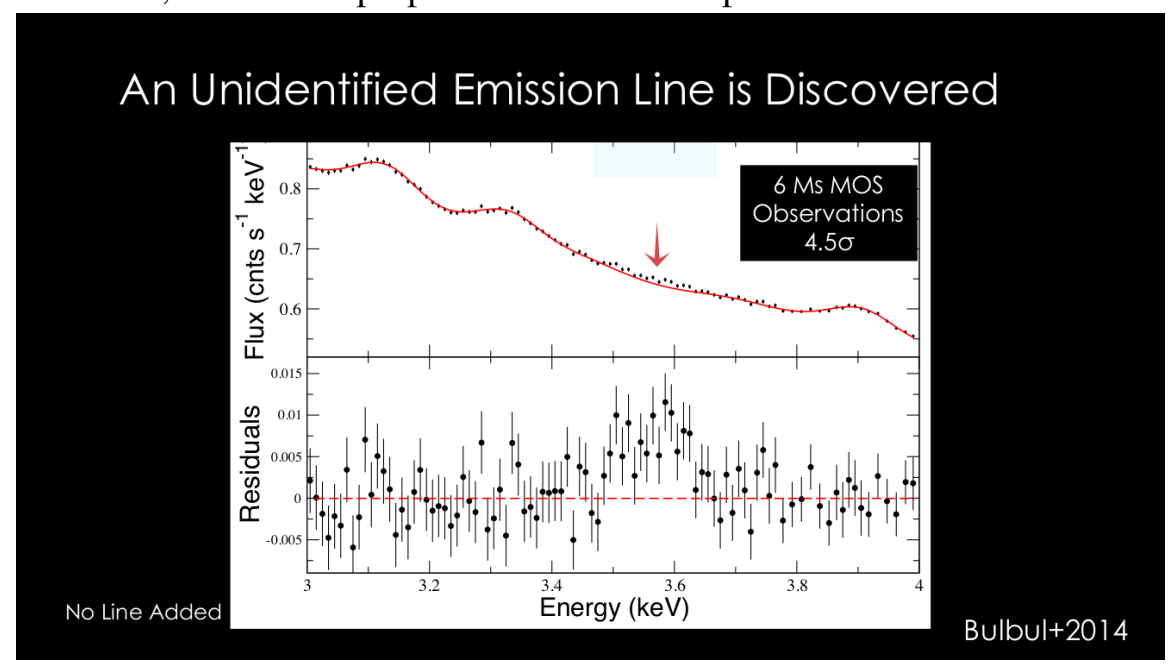

The next figure shows the satellite Hitomi, which did not see the $3.55 \mathrm{keV}$ line from the Perseus

Cluster where it had previously been observed.

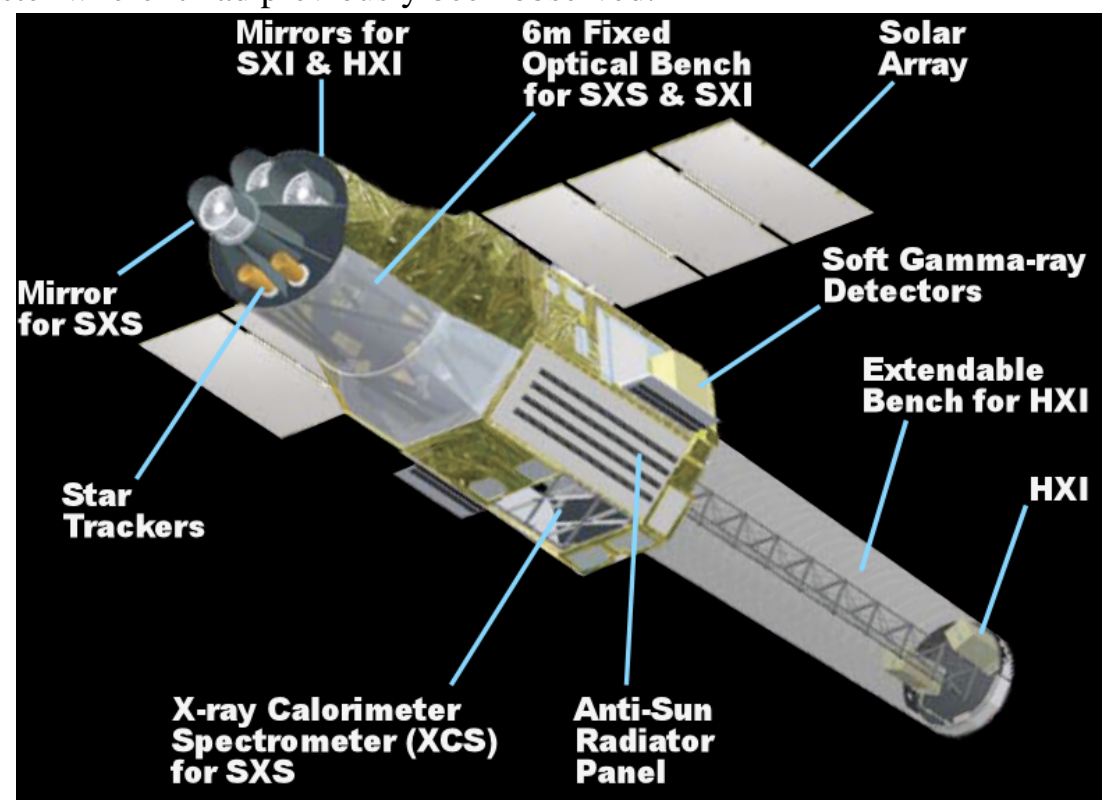


In order to obtain an easy way to compare the prediction of our dark matter pearl model for the flux of the observed $3.55 \mathrm{keV}$ line we shall make use, as an intermediate step, of a model for dark matter being sterile neutrinos by Merle and Schneider [20], a model just made up for making an explanation of the $3.55 \mathrm{keV}$ X-ray line. They end up having fitted the rate of the mysterious line by a life-time of this sterile neutrino, which simply decays to a $3.55 \mathrm{keV}$ photon plus an ordinary neutrino. It is then our strategy to calculate the life-time of the sterile neutrino that would fit or simulate our model. In this way we can essentially just use our model to estimate the one number, the sterile neutrino life-time simulating our model, rather than having to go through each cluster of galaxies or whatever separately with our model. It does of course not mean that we should believe in the sterile neutrino model. The truth is actually that the sterile neutrino model does not really fit so well, and we suspect it is because the sterile neutrino model makes the dark matter $3.55 \mathrm{keV}$ radiation simply be proportional to the amount of dark matter looked at. However, our model with its collisions of pearls or models with the radiation caused by the annihilation of dark matter rather makes the emission of radiation go proportionally to the square of the dark matter density. This is because we need two pearls to get a collision.

\section{"Pedagogical Model" of Merle and Schneider.}

Merle and Schneider propose the simple model that the dark matter consists of "sterile neutrinos" (or other particles) with mass $7.1 \mathrm{keV}$ decaying then very slowly

$$
v_{s t} \rightarrow v+\gamma
$$

Since this is a decay into two massless particles, a neutrino $v$ and a photon $\gamma$, they each get the half energy $3.55 \mathrm{keV}$.

We may represent the experimental rate information by fitting the life-time of this model which we mainly consider pedagogical to describe e.g. the rate of radiation:

$$
\text { "life time" } \simeq 10^{28} s
$$

\section{About the rate of $3.5 \mathrm{keV}$}

The energy release into the $3.5 \mathrm{keV}$ line corresponding to the Merle Schneider model fit of life time $10^{28} s$ means

$$
\begin{aligned}
\text { "Radiation per mass" } & =\frac{\left(3 * 10^{8} \mathrm{~m} / \mathrm{s}\right)^{2}}{2 * 10^{28} \mathrm{sg}} \\
& =0.5 * 10^{-11} \mathrm{~W} / \mathrm{kg} \\
\text { With a dark matter density of } \rho_{D M} & =\frac{m_{\text {proton }}}{3 \mathrm{~m}^{3}} \\
& =10^{-27} \mathrm{~kg} / \mathrm{m}^{3}, \\
\text { "Radiation per volume" } & =10^{-38} \mathrm{~W} / \mathrm{m}^{3} \\
\text { Consider a cube of } 8 \text { kpc sides having volume } & =\left(8 * 3 * 10^{16}\right)^{3} \mathrm{~m}^{3} \\
& =10^{52} \mathrm{~m}^{3}, \\
\text { Power from "galaxy cube" } & =10^{25} \mathrm{~W}(3.55 \text { radiation })
\end{aligned}
$$


This should be compared with

$$
\text { "Luminosity of Galaxy" }=5 * 10^{36} \mathrm{~W}
$$

\section{Criticism of Dark Matter Interpretation of $3.5 \mathrm{keV}$ line}

Jeltema and Profumo [D] criticized the identification of the $3.5 \mathrm{keV}$ line with dark matter, on the grounds that model spectra suggest that atomic transitions in helium-like potassium ( $\mathrm{K}$ xviii) and chlorine $(\mathrm{Cl} \mathrm{xvi})$ are more likely to explain the line. This suggestion has been supported by Phillips, Sylwester and Sylwester [[2]], who point out that the K xviii lines have been observed in numerous solar flare spectra at high spectral resolution with the RESIK crystal spectrometer and also appear in Chandra HETG spectra of the coronally active star $\sigma \mathrm{Gem}$. In addition, the solar flare spectra at least indicate a mean coronal potassium abundance which is a factor of between 9 and 11 higher than the solar photospheric abundance. They suggest that the potassium abundance in the hot plasma in galaxies and galaxy clusters may also have been enhanced compared to the solar photospheric value and thereby explain the $3.5 \mathrm{keV}$ line. However they do not suggest a mechanism to provide such an enhancement.

Although the Hitomi satellite malfunctioned just over a month after launch in February 2016, Hitomi [23] managed to collect enough data to claim the absence of a signal for the $3.5 \mathrm{keV}$ line from the core of the Perseus galaxy cluster. In fact they saw a small dip at $3.5 \mathrm{keV}$.

In spite of the fact that the Draco Dwarf spheroidal galaxy $80 \pm 10 \mathrm{kpc}$ away holds so much dark matter that its ratio of mass to luminosity is $440 M_{\text {sun }} / L_{\text {sun }}$, it was found in [24] that it has no $3.5 \mathrm{keV}$ line with a $99 \%$ confidence 20 times smaller than expected, if it were radiation simply from dark matter decay. However another analysis [25] of the Draco dSph data does find a positive signal with a low $2 \sigma$ significance, as shown in the next figure of line flux against line energy in $\mathrm{keV}$ where filled rectangles show the range of fluxes predicted from previous works.
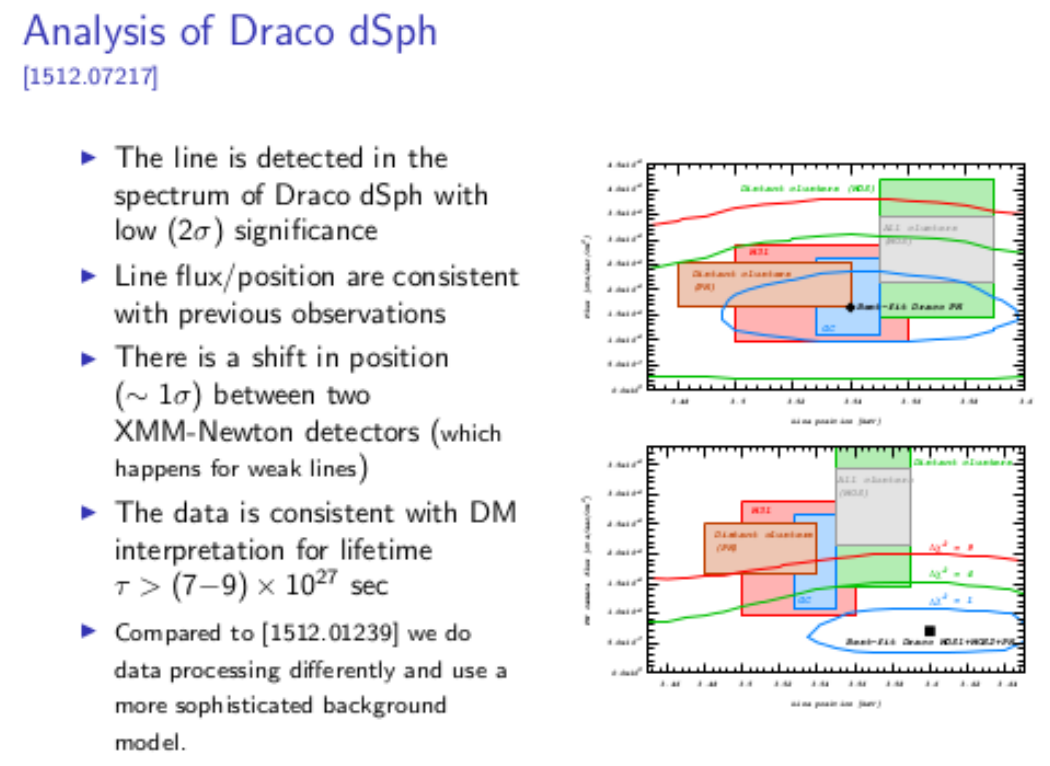

\section{Any Hope for Rescuing a Connection to Dark Matter?}

There is clearly conflicting evidence on the existence of the $3.5 \mathrm{keV}$ line. A more detailed review of the observation of a $3.5 \mathrm{keV}$ line in the spectra of galaxies and galaxy clusters is given 
by Iakubovskyi [26]. In the next figure we give a summary of the positive and negative evidence for the decaying dark matter model for this line.

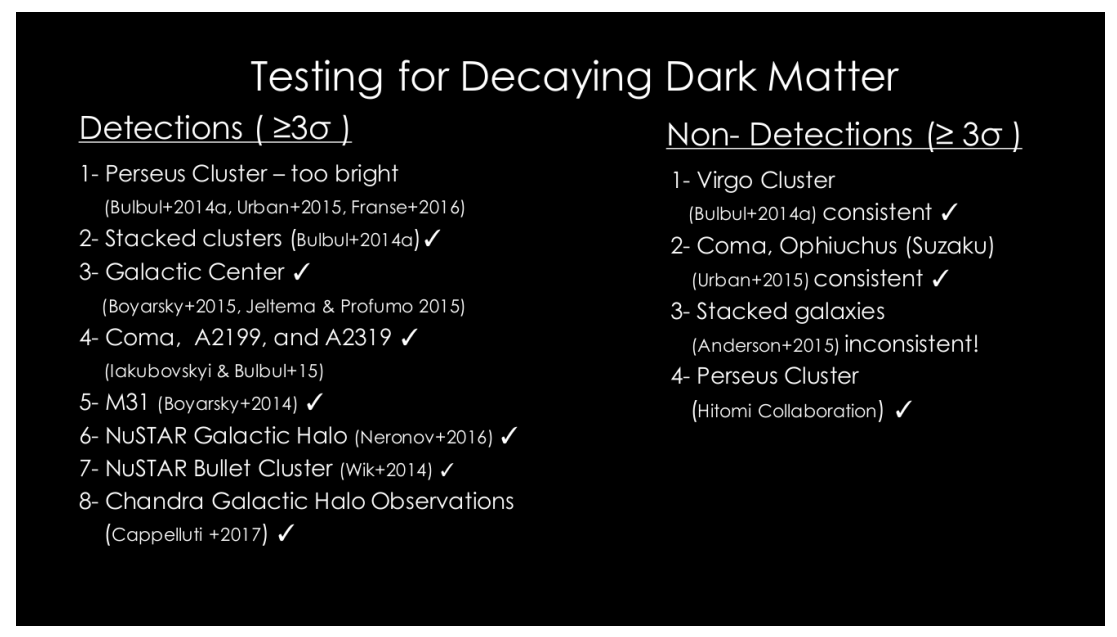

The morphology of the $3.5 \mathrm{keV}$ line was studied by Carlson, Jeltema and Profumo [L]] using a broad sample of continuum emission models. They found that the clumped nature of the residual emission line in the Perseus cluster is difficult to reconcile with the much smoother distribution expected from dark matter, as is the radial profile which has a much sharper gradient at the edge of the core than what expected from a decaying dark matter profile. Similarly, in the case of our Galactic Centre, the azimuthal and radial distributions of the residual signal at $3.5 \mathrm{keV}$ is found to be strikingly different from the prediction from dark matter decay.

It therefore appears that, if the line $3.5 \mathrm{keV}$ should come from dark matter, it is produced by some interaction so that the line signal gets produced mostly from regions with lots of e.g. ordinary matter, that e.g. could throw some radiation on the dark matter. Alternatively the dark matter could interact with itself, as in the collision of two of our dark matter pearls.

In the next figure we present the flux of the $3.5 \mathrm{keV}$ line from the Galactic Centre, the Perseus cluster outskirts, the Andromeda galaxy M31 and the blank sky (Milky Way halo) data set [ए耳] as a function of the dark matter projected mass. Diagonal lines show the expected behaviour of a decaying dark matter signal for a given dark matter particle lifetime. The vertical sizes of the boxes are $\pm 1 \sigma$ statistical error on the line's flux - or the $2 \sigma$ upper bound for the blank sky data set. The horizontal sizes of the boxes bracket the scatter of the dark matter density profiles in the literature. The blue shaded regions show a particular Navarro-Frenk-White profile of the Milky Way [28], its horizontal size indicates uncertainties in galactic disk modeling. The lifetime $\tau_{D M} \sim(6-8) x 10^{27}$ sec is consistent with all the data sets. 


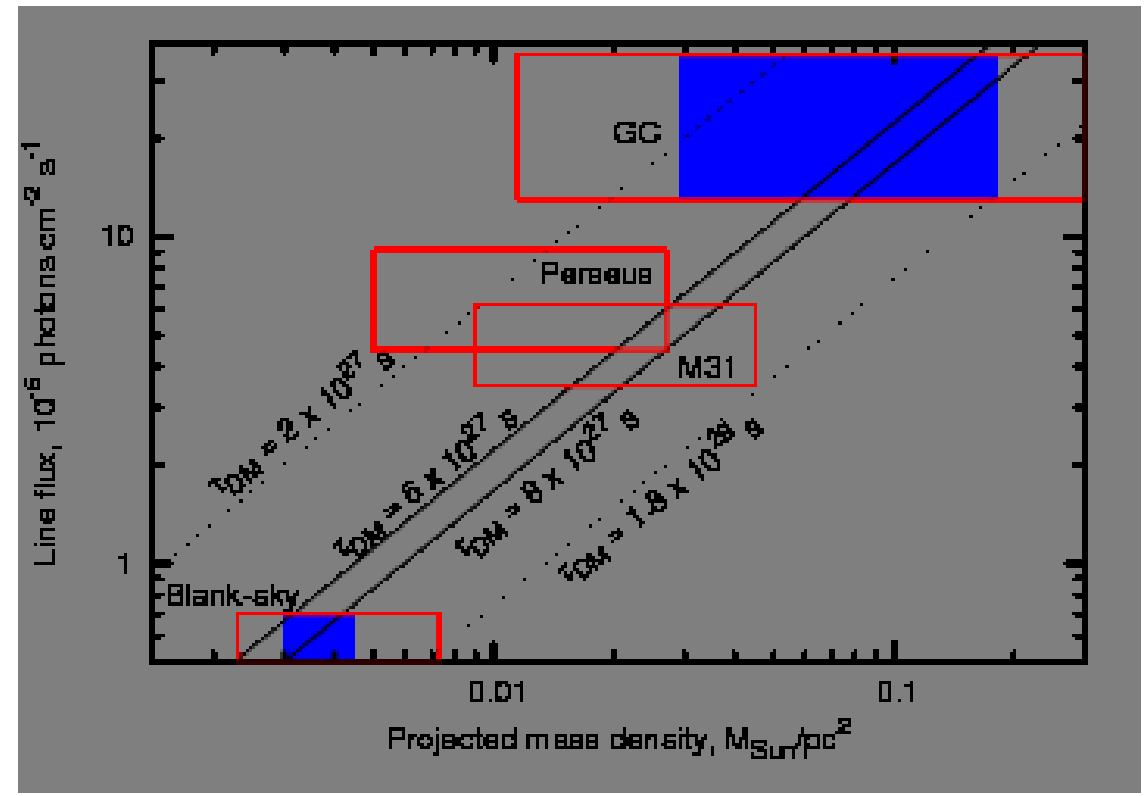

\section{MPP: New Law of Nature: Multiple Point Principle.}

There are several phases of vacuum (having relativity principles) and they all have very very small energy densities (like the astronomically determined one for our present vacuum).

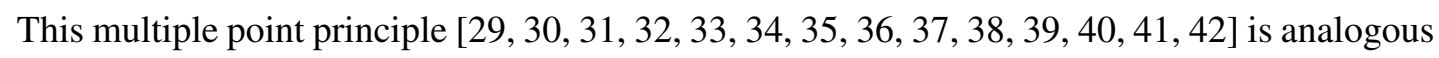
to having a specific temperature - in a microcanonical ensemble - when there are say both water and ice present (as in the figure below).

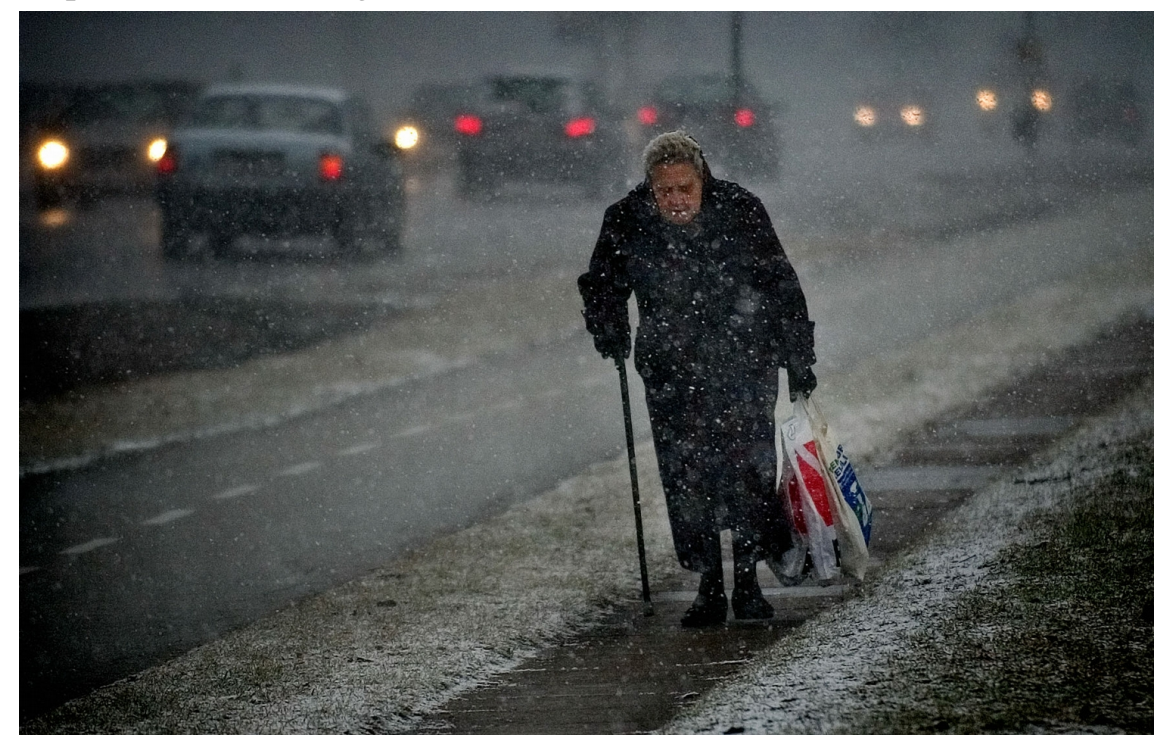

The analogy between the triple point in the vapour-water-ice combination in the bottle illustrated below at temperature and pressure $(T=273.16 \mathrm{~K}, P=0.6117 \mathrm{kPa})$ and the "Multiple Point Principle" is as follows: 


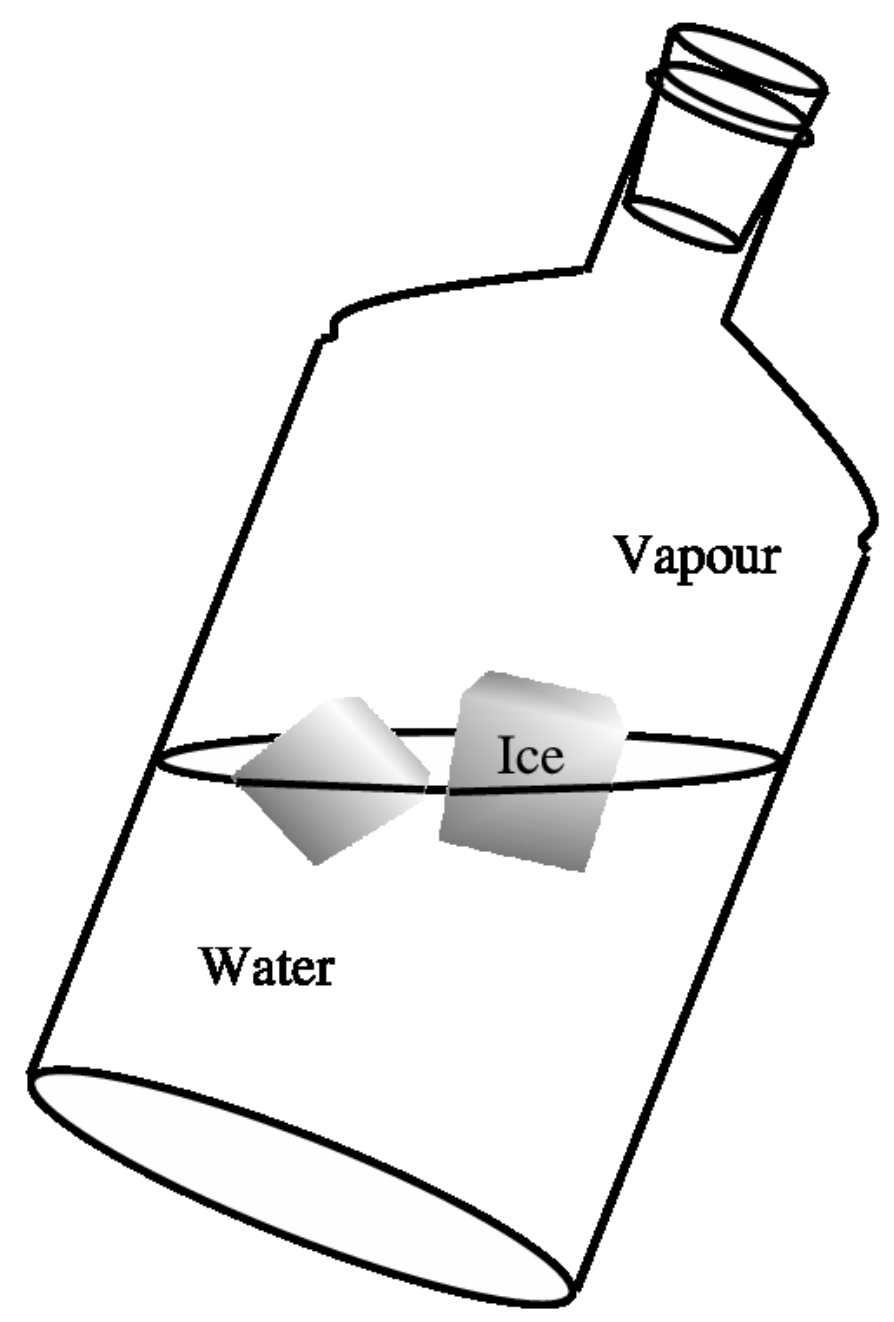

- The intensive quantities, temperature $T$ and pressure $P$, are analogous to the coupling constants and the parameters, such as $g_{t}, \alpha=\frac{e^{2}}{4 \pi}$, etc.

- The extensive quantities such as the energy $E$ of the contents of the bottle, the amount (e.g. measured in mol) of water-molecules $N$ and the volume $V$ are analogous to some integrals, which could include

- The space-time volume $=$ lifetime of universe multiplied by its volume.

- An integral over the square of the Higgs field = the average value of the Higgs field squared and multiplied with the just mentioned space-time volume.

- But it could be something similar based on other fields.

\section{We PREdicted the Higgs mass! [32, [33, 47, 148, 49, 50, 5]]}

Using the Multiple Point Principle, we require that the coupling constants - as for example the top quark Yukawa coupling related to the top quark mass - and the other parameters in the field theory (in this case the Standard Model) - such as the Higgs mass squared - are fine-tuned to take on such values that the existence of degenerate vacua having the same energy density is ensured. 
In addition to the usual vacuum with a Higgs vacuum expectation value of $246 \mathrm{GeV}$, we obtained another vacuum degenerate with it but having a very large Higgs field expectation value of order the Planck scale $\sim 10^{18} \mathrm{GeV}$. As a consequence a fine-tuned value for the Higgs mass squared is obtained and thus two of us (CDF and HBN) predicted - long before the Higgs boson was found the Higgs mass $m_{H}$. Our prediction $m_{H}=135 \pm 10 \mathrm{GeV}$ (and the top quark mass of $174 \pm 5 \mathrm{GeV}$ ) is captured in a painting of one of us together with the Danish finance minister Mogens Lykketoft (on the blackboard behind the head of Lykketoft).

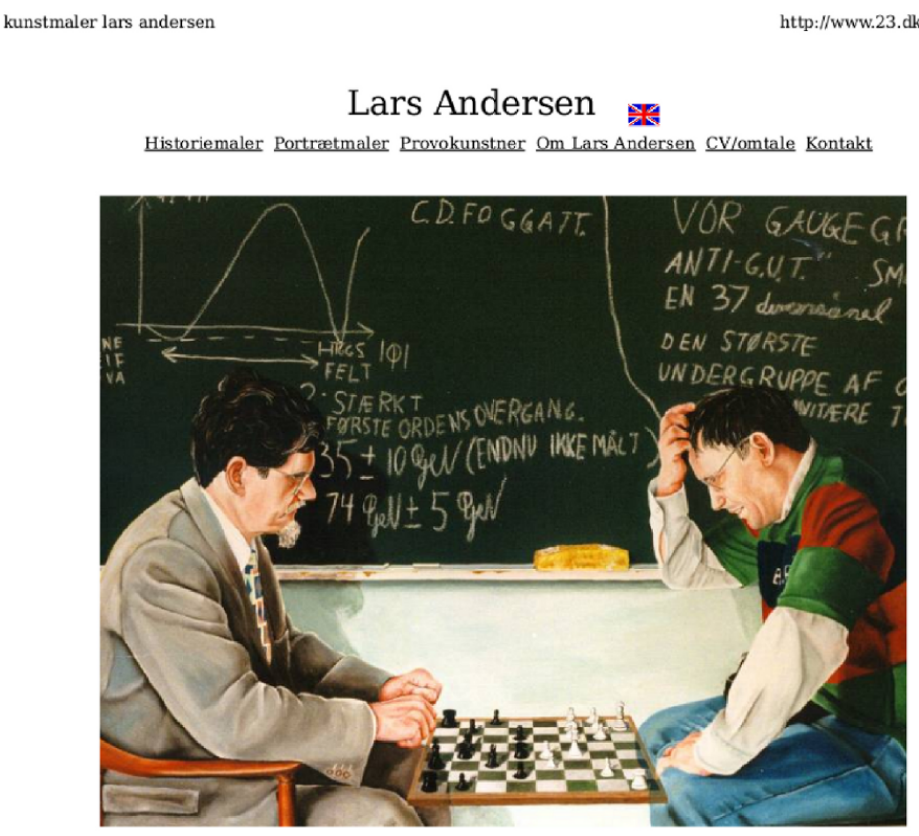

"Skak" (Mogens Lykketofte og Holger Bech Nielsen)

\section{Tilhører Frederiksborg Museet}

Hœnger i moderne samling i det Nationalhistoriske Museum på Frederiksborg Slot.

Bragt i årbogen "Dansk Kunst 1998", Jyllandsposten, Politiken, BT, Ud og Se,

Berlingske Tidende, Ekstra Bladet, Kristeligt Dagblad, Att for Damerne.,

Frederiksbory Amts Avis, DR1, DR2, TV Lorry, Kunstavisen Billed Bladet....

Trykt som plakat $50 \times 70 \mathrm{~cm}$.

The existence of the Higgs boson was confirmed in 2012 and the experimental mass is $m_{H}=$ $125.09 \pm 0.21$ (stat.) \pm 0.11 (syst) $\mathrm{GeV}$ deviating by only one standard deviation (of our theoretical uncertainty in 1995) from our MPP prediction. In a slightly modified version of MPP, called Meta-MPP, requiring just meta-stability rather than degenerate vacua, we obtained together with Y. Takanishi [52] $m_{H}=121.8 \pm 10 \mathrm{GeV}$. The instability, metastability and stability regions in the Higgs mass versus top quark mass plane are given below. 


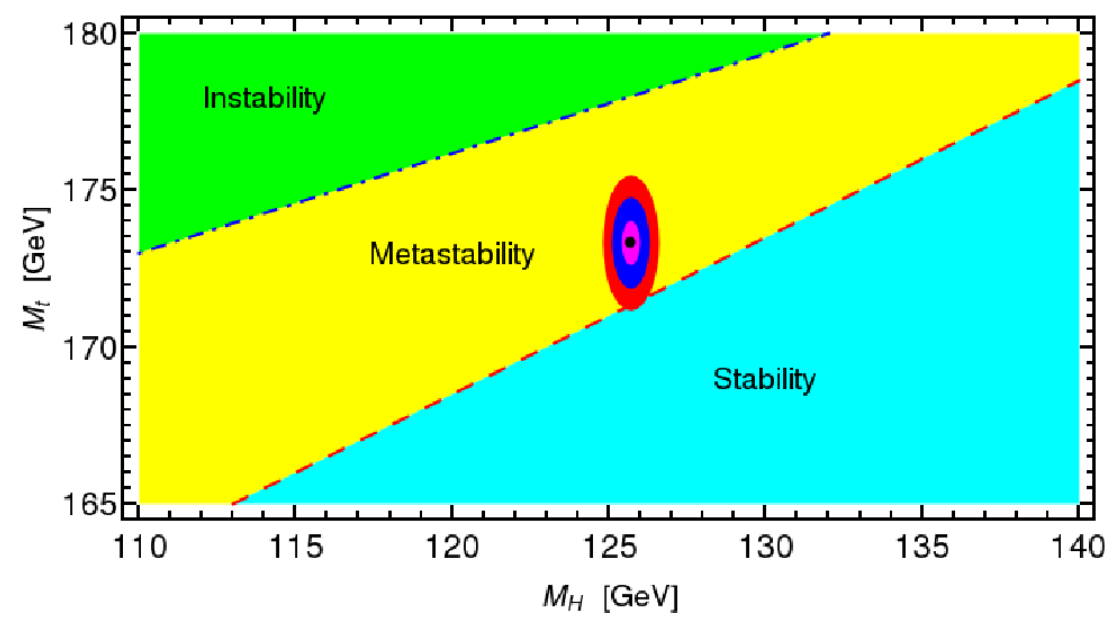

\section{Three Agreeing Fits of the Bound State Mass:}

We have now introduced two vacua, the condensate vacuum inside our dark matter pearls and the high field vacuum in our calculation of the Higgs mass, degenerate with the usual Standard Model vacuum. The condensate vacuum is supposed to contain a lot of strongly bound states $F$ distributed smoothly in space, each bound state consisting of 6 top quarks and 6 anti-top quarks. As discussed in the Proceedings of the Corfu Summer Institute 2016 [53], we can use these two vacua and the bag model to estimate the mass of this bound state $F$.

$$
\begin{aligned}
m_{F}(\text { from "high field vacuum") } & \approx 850 \mathrm{GeV} \pm 30 \% \text { with } \sim 2 \text { factor } \\
m_{F}(\text { from "high field vacuum" }) & \approx 710 \mathrm{GeV} \pm 30 \% \text { without } \sim 2 \text { factor } \\
m_{F}(\text { "condensate vac." }) & \approx 692 \mathrm{GeV} \pm 40 \% \\
m_{F} \text { ("bag estimate") } & \approx 5 m_{t}=865 \mathrm{GeV} \text { (very uncertain). }
\end{aligned}
$$

The $\sim 2$ factor was to take into account the contribution of neglected diagrams in the calculation.

\section{The Cold Dark Matter out between the Stars in Space}

Our colleagues think that the dark matter, needed in order that stars can run so fast around the Galaxy as they are measured to run, cannot be obtained, if the Standard Model is the final theory. One needs at least one extra type of particle, that can make up the dark matter! Only two of us CDF and HBN - have a theory, in which it is possible - although in an a somewhat complicated way - to get the dark matter in the Standard Model alone ! Let us, however, admit that we need a special fine-tuning-principle, which requires the Standard Model couplings taking on such values that several vacua/phases of empty space appear with the same energy density. The couplings thus have very special values, or rather relations between their values.

On the following figure one sees a galaxy - that could be our own or another one - together with a curve illustrating the velocity of the stars in their essentially circular motion around the galaxy centre as a function of their distance to this centre. 
The curve giving the actual measured - by the Doppler effect - velocities starts at zero at zero distance, i.e. at the centre, and grows up approximately linearly at first, but then flattens out and approximately stabilizes at a circulation velocity $\sim 200 \mathrm{~km} / \mathrm{s}$. The other curve - the red one - gives the theoretical expectation using the usual Newton's law of gravitation together with the amount of matter calculated according to the observed densities of stars and gas (etc.). This curve follow the observed star-velocity curve for small distances, but not only flattens off, but rather falls down again and goes to zero velocity for very large distances. The discrepancy between the two curves means that Newton,s law of gravity is not working at all for distances of galactic size, or that there is some sort of matter, which the astronomers estimating the mass in the stars, gas etc. have not taken into account!

This extra matter is needed to have a much less concentrated distribution than the stars and gas etc. which were visible and thus included in the estimate of the astronomers to produce the expected curve falling so much more rapidly off at long distances than the observed star velocities. But this extra matter should not be visible. It is this extra matter dreamt up to make Newton's gravitation law to work which we call "dark matter".

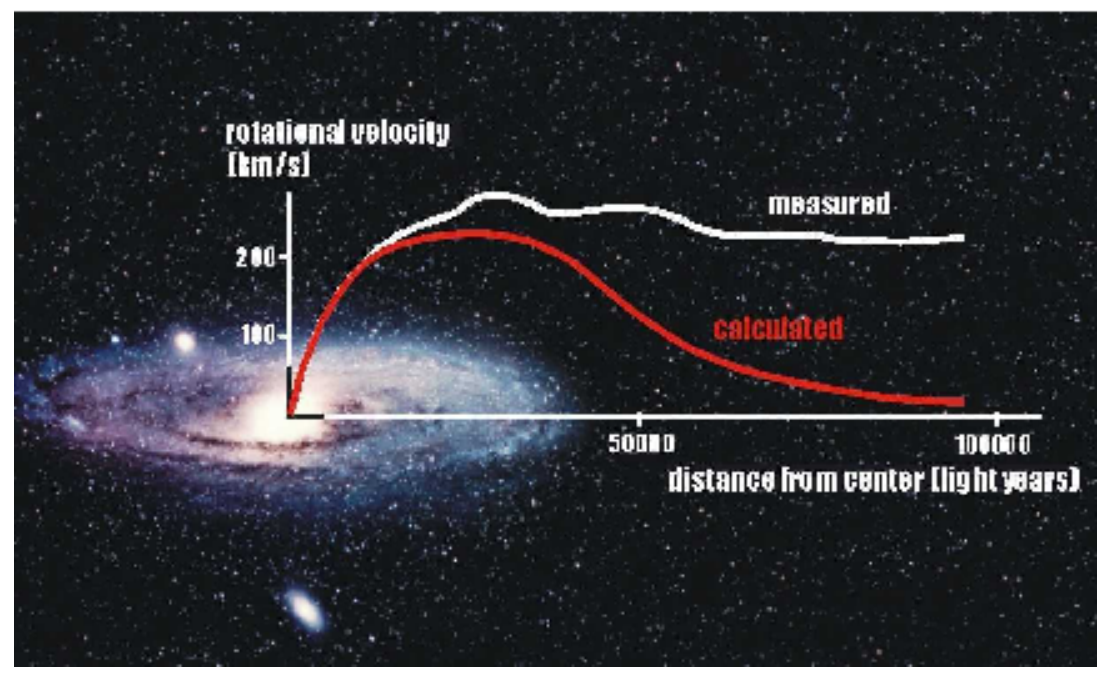

On the next picture we see Zwicky, who was the first to observe effects of dark matter (or perhaps of violation of Newton's law): 


\section{Fritz Zwicky, Opdager af Mørkt Stof}

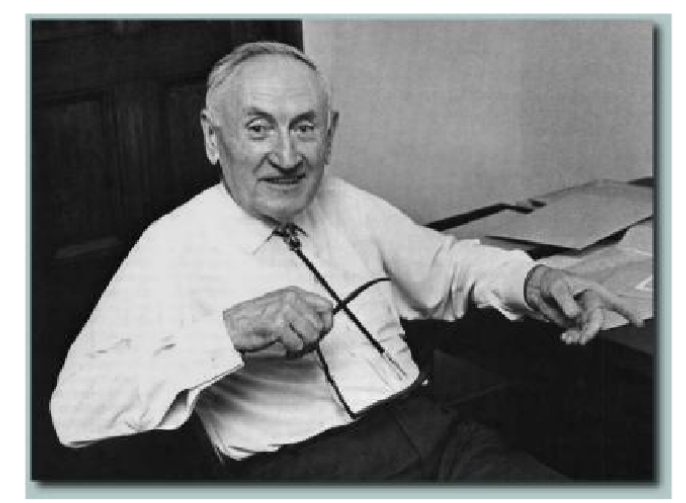

There are many attempts in high energy physics to invent models for what the dark matter could possibly be. Mostly physicists speculate that it is some new particle, not one of the ones in the Standard Model. If they have a small mass, as e.g. the weight of an atom, and interact weakly they will at best be observable on earth by sophisticated counters. What is important for some speculated material to be useful as the dark matter at all is that the interaction per $\mathrm{kg}$, so to speak, is small enough.

If, however, now as in our - two of us CDF and HBN - model the dark matter consists of 100000 ton to a million ton heavy pearls, then such pearls would still interact sufficiently weakly to be applicable as dark matter even if they had normal interactions with matter as if they were $\mathrm{cm}$-sized pearls, such as we indeed assume them to be.

To deliver the correct density for the dark matter, such hundred thousands of ton heavy pearls would have to be separated by distances of the order of astronomical units or many times the distance to the moon. They would, according to our suggestive numbers, hit the earth approximately once every two hundred years.

But a pearl of million ton mass and such a small $\mathrm{cm}$-size hitting the earth would penetrate deeply, thousands of km's into the earth. It would heat up dramatically the tube of earth along its path through the earth, and material from the heated up tube of earth would spit out to the surface of the earth somehow. This would look like volcanic activity. We actually believe that one should identify such eruptions, due to the fall of one of our pearls, with what geologists see as kimberlite pipes [3], 54, [55]. This is a kind of volcano with the type of stone called kimberlite filling the tube. Typically there are also diamonds buried in the kimberlite. We therefore believe that once upon a time it was the impact of one of our pearls of dark matter, that caused the volcanoes, which are now often used as diamond mines. The next picture illustrates such a diamond mine and thus presumably the result of a once fallen pearl of dark matter: 


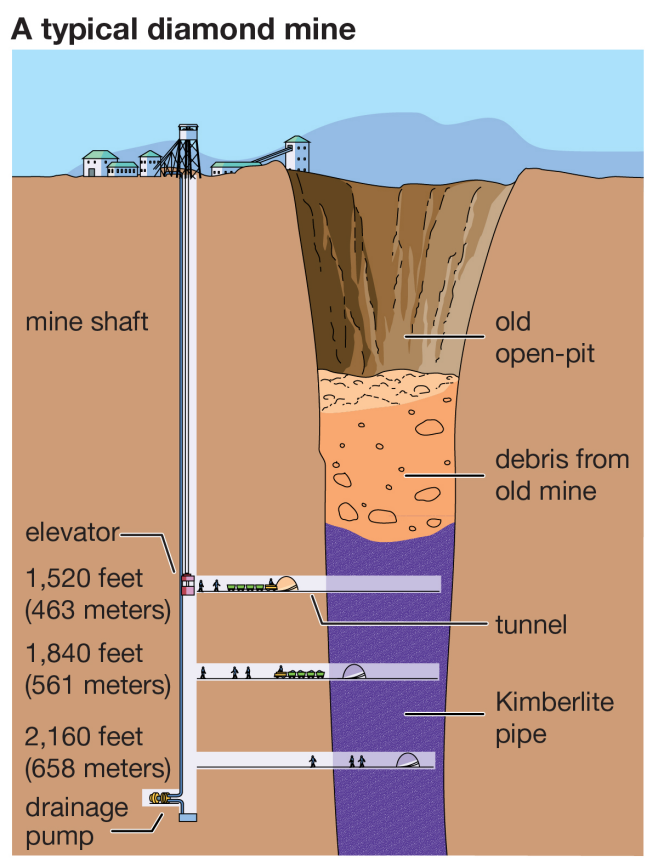

The next figure should illustrate an overview of what we imagine: Out in space our pearls fly around with hundreds of $\mathrm{km} / \mathrm{s}$ velocity. From time to time - about every 200 years - one of then hits the earth and penetrates deeply into it. Thereby a volcano is created, giving a kimberlite pipe or several pipes.

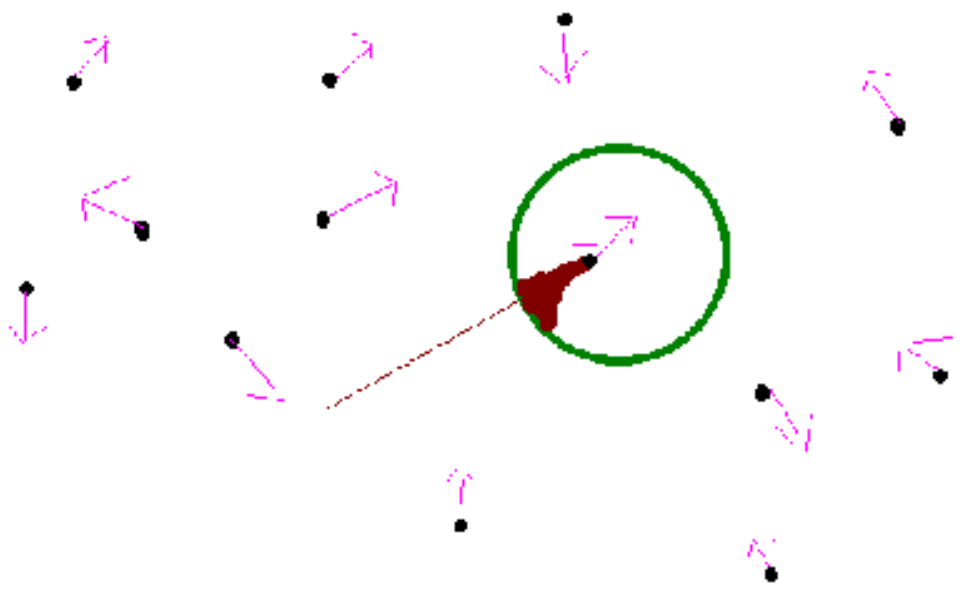

It is told - by geologists - that a sign for such a kimberlite pipe volcano is a little ( $\sim 1 \mathrm{~km})$ round lake. The next figure shows such a "little round lake": 


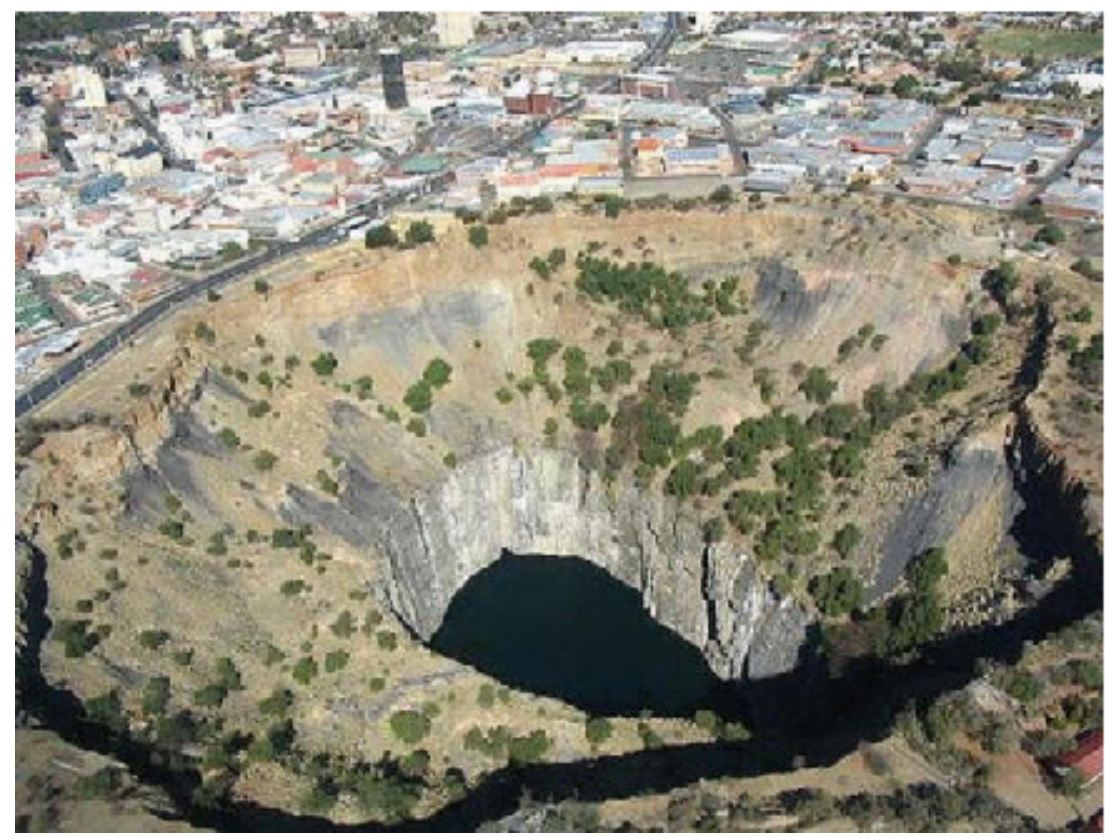

The next figure is a map illustrating that there is a region in Greenland with many such kimberlite pipes:

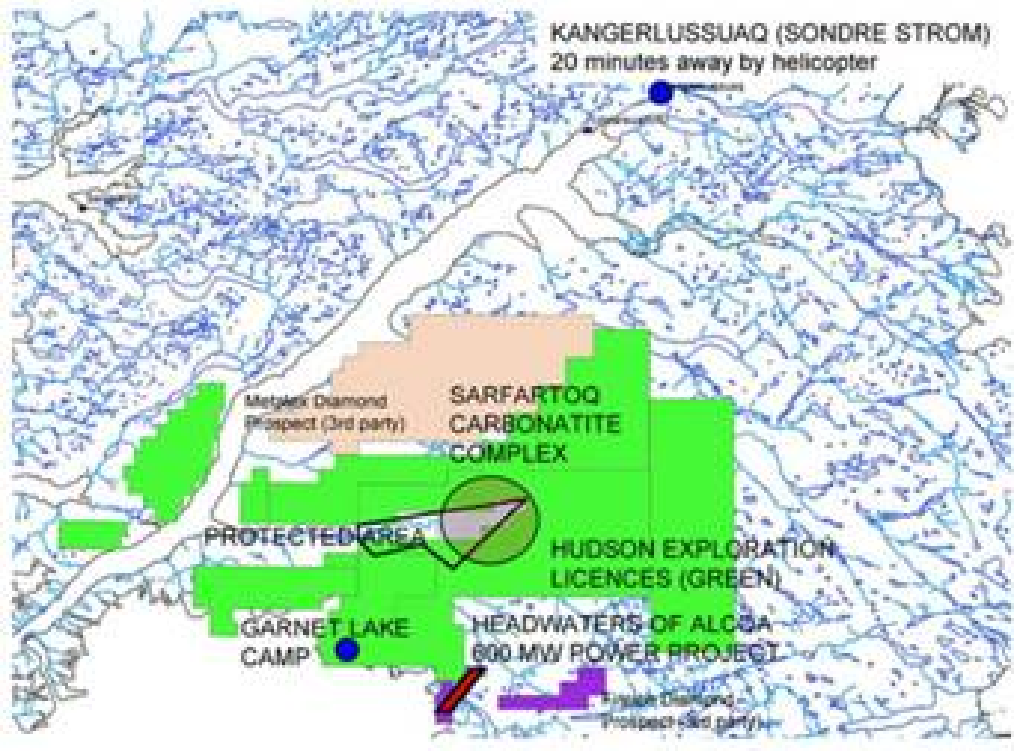

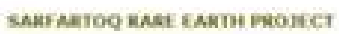

The next figure is an illustration meant to explain that actually diamonds are only stable under very high pressure. So if diamonds are stable deep in the earth and come up slowly, they will become graphite on the way up. So, unless there is some mechanism by which the diamonds can come up very quickly and avoid lying for a long time in the heat under lowering pressure, there would be no diamonds at all for human beings to get hold of. Thus in our picture, it is only due to the dark matter pearls that humans have ever got accustomed to diamonds at all:

For us who care for if these kimberlite pipes are indeed due to some impact at all or perhaps more likely some genuine volcanic activity having its origin deep inside of the earth, rather than 


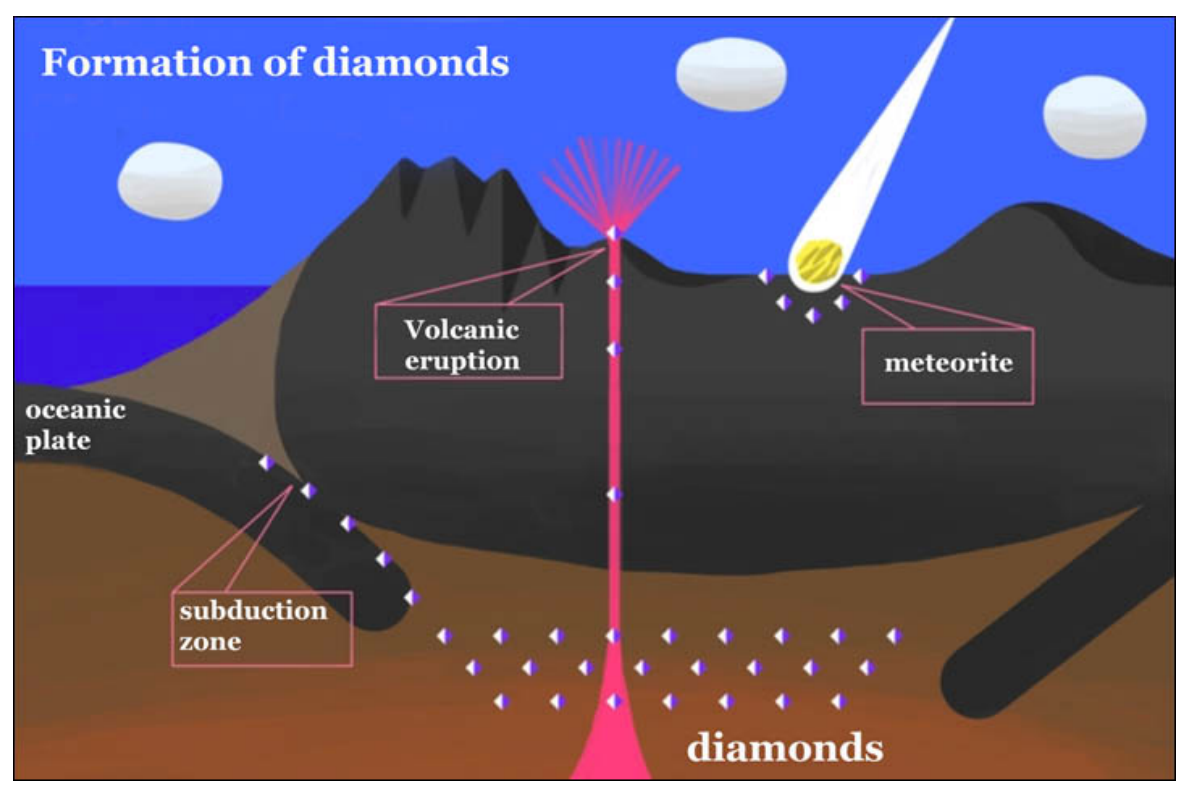

being due to an impact of some highly dense mysterious piece of matter, it is interesting to consider the structure of such a volcanic tube as shown below:

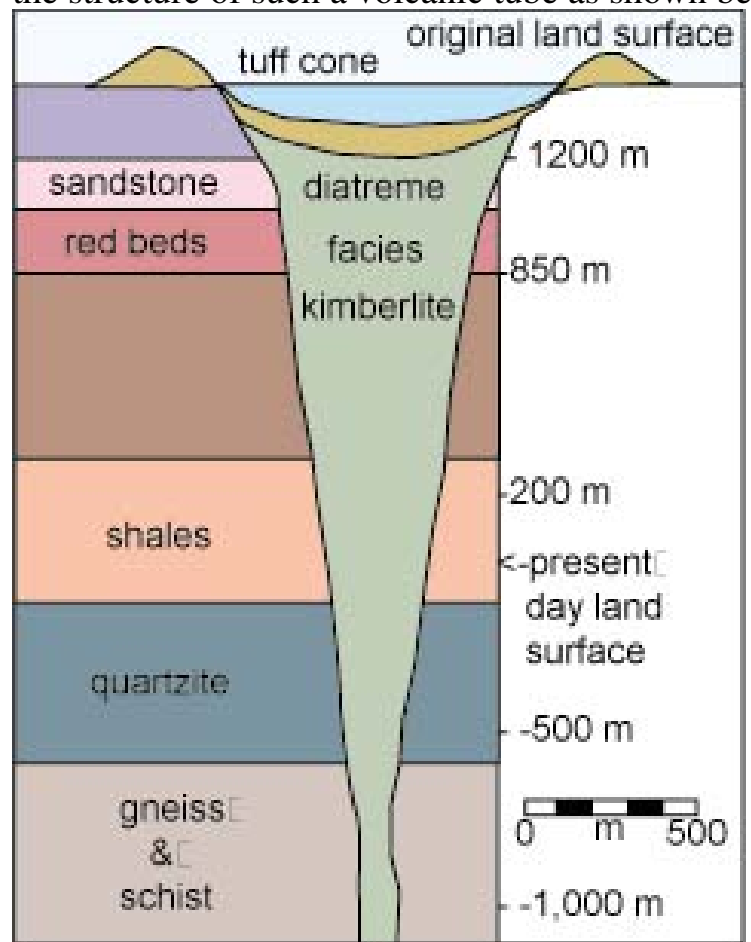

We should also point out a special property of the pearls of dark matter in our detailed model [B]]: when a pearl is hit by a neutron, this neutron gets absorbed together with the production of about $10 \mathrm{MeV}$ heat energy: 


\section{Pearl produce about 10 MeV energy per neutron caught}

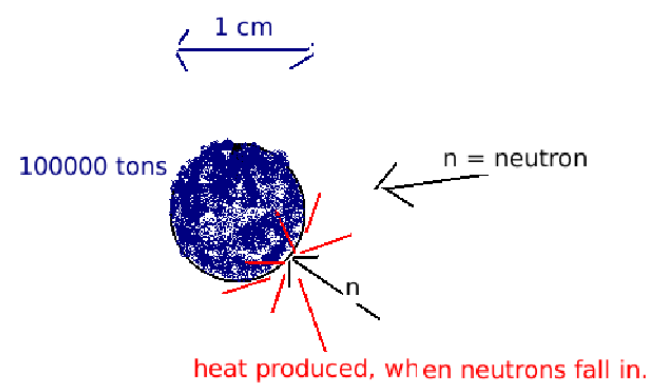

\section{Supernova development}
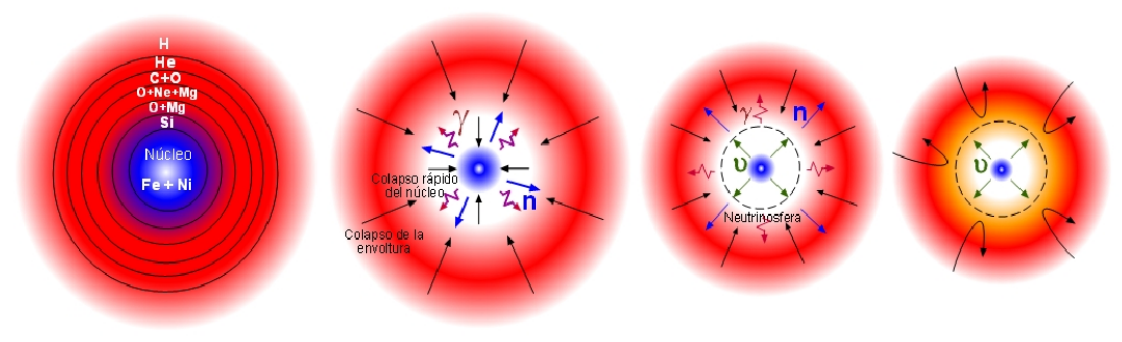

This property of our pearls of being so good at swallowing up neutrons makes them begin to swallow a major part of a neutron star at the end when, in a supernova explosion, a neutron star is formed. A very short review of the development of a star ending in a supernova explosion is put forward in the figure above. In our article [56] we argue that the presence of our pearls and their eating of neutrons is actually both helpful for really making the supernova explosions emitting lots of energy come about and, also, for explaining an otherwise very mysterious observation concerning the supernova 1987a in the Big Magellanic Cloud, for which two large bursts of neutrinos were observed to be emitted from the supernova with a time difference of about 5 hours. Indeed we estimate there to be such two outbursts with a few (actually 14 in order of magnitude) hours interval 
between them.

\section{Dark Matter Collisions: Collisions of Our Pearls as Source of Energy for e.g. the $3.5 \mathrm{keV}$ line.}

Somewhat analogous to annihilation, in our pearl model a lot of energy can be released when the dark matter particles meet/collide:

We expect them to unite and then the surface/skin to contract and thereby release energy; in fact about the Einstein energy of a tenth of the mass of the pearl.

The following figure illustrates what happens in the very seldom cases when two of our pearls collide. These two pearls are two droplets of the new "condensate vacuum" type of vacuum (containing ordinary matter). Like two water droplets will typically unite when hitting each other, we expect our pearl droplets to unite. But thereby the surface can be rearranged and will arrange itself to be smaller in area than the sum of the surface areas of the two colliding pearls. Since in our model we assume the surface tension to be given order of magnitudewise in a dimensional argument, using as the typical mass the top-quark or almost equivalently some weak interaction scale, the surface tension is huge compared to the usual chemistry scale. Therefore a very large release of energy is possible in the collision.

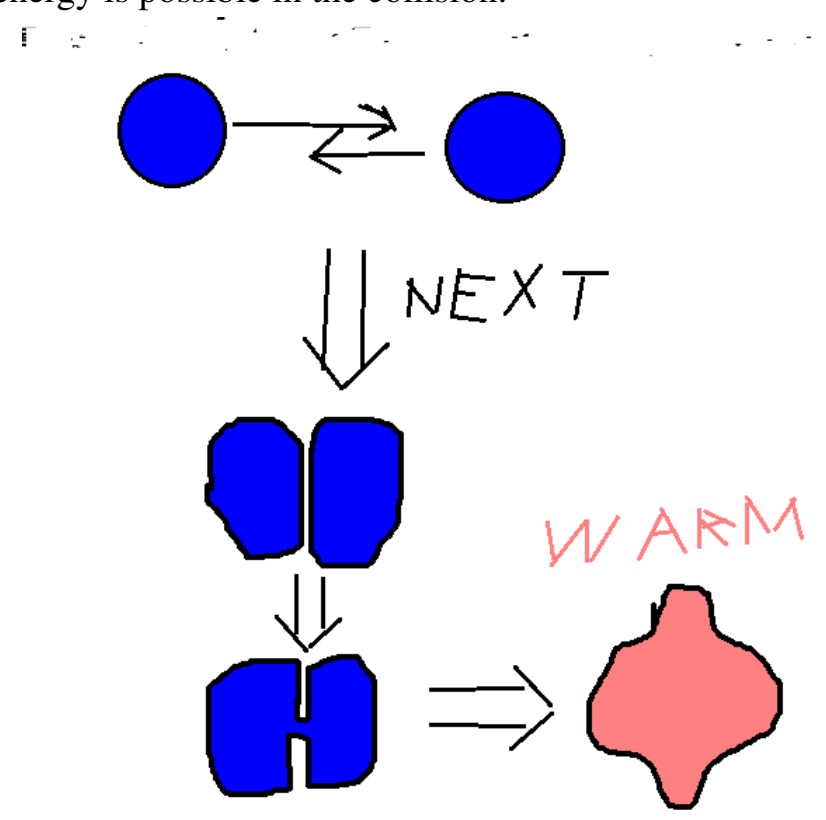

\section{Self Interaction of Our Pearls}

We fitted our model parameters so that the rate of the earth being hit by one of our dark matter pearls is about once every 100 years or 200 years.

Now the ratio of the radius $r_{\text {pearl }}=0.6 \mathrm{~cm}$ of our pearl to the radius of the earth is

$$
\begin{aligned}
\frac{r_{\text {pearl }}}{r_{\text {earth }}} & =\frac{0.6 \mathrm{~cm}}{6000 \mathrm{~km}} \\
& =10^{-9}
\end{aligned}
$$


giving the ratio of areas to be

$$
\frac{\text { area }_{\text {pearl }}}{\text { area }_{\text {earth }}}=\left(10^{-9}\right)^{2}=10^{-18}
$$

\section{Estimating Rate of 3.5-line from Pearl Collisions}

Because the area for hitting another pearl is $10^{-18}$ times that for the earth, a pearl - a selected one, we think of - will hit another pearl every:

$$
\text { "hitting interval" }=100 \text { years } * 10^{18}=10^{20} \text { years }=3 * 10^{27} \mathrm{~s} .
$$

When a collision between two of our pearls occurs an energy of the order of the energy in the bubble surface is released, and likely a large fraction of that becomes excitons and thereby gives the $3.5 \mathrm{keV}$ radiation.

\section{Ratio of Surface to Bulk Energy}

The surface tension of our pearls is supposed to be of the order of magnitude as given by the weak interaction physics, say given in terms of $\mathrm{W}$-masses by dimensional arguments. By the unification of two pearls after a collision the total surface area for the uniting bubbles is reduced by of order unity. Taking crudely the weak interaction length scale to be $10^{-18} \mathrm{~m}=10^{-16} \mathrm{~cm}$ and the energy to be $100 \mathrm{GeV}=100 * 10^{9} * 1.6 * 10^{-19} \mathrm{~J} \sim 10^{-8} \mathrm{~J}$, the energy in the tension of the pearl surface becomes

$$
\begin{aligned}
\text { "surface energy" } & =\left(10^{16}\right)^{2} * 10^{-8} \mathrm{~J} \\
& =10^{24} \mathrm{~J} \\
& \sim 10^{24} / 10^{17} \mathrm{~kg}=10^{7} \mathrm{~kg}
\end{aligned}
$$

This is about $\frac{1}{10}$ of the mass of the whole pearl $10^{8} \mathrm{~kg}$.

Simulated Life- time $10^{28} s$.

Since about one tenth of the Einstein energy of the pearl sits in the surface tension and is released by the collision of two pearls, the life time of a simulating sterile neutrino model particle would be 10 times the "hitting time" $\sim 10^{27}$ s, i.e a $10^{28} s$ simulated life time. This agrees well with the life time $\tau_{D M} \sim(6-8) \times 10^{27}$ s for decaying dark matter extracted from the data!

The Energy Comes in Bunches of $\sim 10^{7} \mathrm{~kg} * c^{2}$

With our story that the dark matter consists of pearls with a surface energy getting released when two of them collide and unite to one, we have got the dark matter pearls to function as bombs, releasing $10^{5}$ times more energy than from one from just colliding with the earth. By a pure collision of one dark matter pearl with the earth, we get the energy of the event in Tunguska, which knocked down the trees in a region of order of $70 \mathrm{~km}$ extent. However with the unification of two pearls we get about 100000 times as much energy released due to the enormous surface tension!

The temperature may raise to $\sim 50 \mathrm{MeV}$ and corresponding $\gamma$-rays would be emitted, a candidate for a gamma-ray burst?

That the radiation from the dark matter in this way comes in pulses, may be experimentally accessible. 


\section{Homolumo: Homolumo-gap-effect}

\subsection{General idea of homolumo-gap-effect.}

What we here call "homolumo-gap-effect" is the effect of increasing or producing the gap between the highest occupied (ho) single electron state (=molecular orbit=mo) and the lowest unoccupied (lu) single electron state (mo), originating from the electrons acting back on say the positions of the ions in the material considered. One shall have in mind that increasing the homolumo-gap lowers the energy of the system of single electrons, and thus the ions are driven in the direction by which they can increase this gap.

\subsection{Crude Ideas about Homolumo gap Effect in Very Dense Matter (inside our pearls)}

Expectations for very highly compressed matter:

- We expect that the kinetic energy of the electrons will dominate over the potential energy. (The inverse lattice momentum scales inversely with lattice constant $a$, i.e. as $1 / a$, and thus the kinetic energy (for e.g. a quasi-momentum a certain fixed fraction of the umklapp momentum) with the inverse square:

kinetic energy $\propto 1 / a^{2}$,

while the

potential energy $\propto 1 / a$.

- Still if the material is a glass, say, a homolumo gap effect could be there, close to the fermisurface.

- We expect the homolumo gap to be crudely of the order $p_{\text {fermi }} \alpha^{2}$, where $\alpha$ is the fine structure constant and $p_{\text {fermi }}$ is some characteristic momentum for the electrons, the fermi momentum say.

\subsection{Simple Statistical Calculation of the Homolumo-gap in Matrix Model}

I. Andric, L. Jonke, and two of us (DJ and HBN) calculated the homolumo gap in a relatively simple matrix model, although it has both quenched random and quantum mechanical adjustable contributions to the single fermion hamiltonian.

Our - Andric, Jonke, Jurman, HBN - Matrix Model for Homolumo-gap Effect []]

The model, we developed, mostly was one with dynamical matrix elements for the electron Hamiltonian, denoted as a matrix $\mathscr{M}$, the elements of which are quantum mechanical bosonic variable, but which in addition are quenched random in the sense that they have their equilibrium points corresponding to a matrix $\mathscr{M}_{0}$, which is taken to be a quenched random matrix (being set at the outset before the adjustment to lower the energy of the dynamical part of $\mathscr{M}$ ). We consider it not so important, but mainly for easier thinking, we take that the matrix $\mathscr{M}$ (and thus of course also $\mathscr{M}_{0}$ ) to be of finite order. But if we do so by, for instance, only including into the electronic state system the states from a few bands, then the order of $\mathscr{M}$ must be proportional to the extensive quantities like the volume or the mass of a macroscopic piece of material, if we want to apply our formulas to such a macroscopic piece of matter. 
Our specific model has a Hamiltonian consisting, of course, first of the energy of the system of what we call fermions in our paper, but which we here call electrons, being simply

$$
\mathbf{H}_{\text {ferm }}=f^{\dagger} \_\mathscr{M} f \mid .
$$

\subsection{Adjusting the $\omega$ 's of Our Matrix Model to Realistic Materials}

There is one little problem with our general calculation in the work I. Andric et al [ए]], namely that for a macroscopic piece of material the number of single electron states, $\mathrm{N}$ say, in a specified set of bands will grow proportionally to the volume $\mathrm{V}$ and thus the number of matrix elements will grow like $N^{2} \propto V^{2}$; but now there cannot reasonably be a number of degrees of freedom of the material growing like this square. Any number of degrees of freedom in the material should grow only as $N \propto V$ and NOT like the square!

\section{How to Approximate Functions of Some Variables by Harmonic Oscillators}

In the work by Andric et al. we calculated as if the matrix elements of the single fermion Hamiltonian were independent harmonic oscillators - each matrix element $\left(M-M_{0}\right)_{i j}$ was an independent degree of freedom variable $q_{l}^{(A)}$, where then of course the index $l$ run over $N^{(A)}=N^{2}$ values, where $N$ is the order of the matrix $M_{i j}$ for the single fermion (say electrons in case of ordinary materials) states relevant (we imagine the very highest energy electron states thrown out as an ultraviolet cut-off, say).

But now these $N^{(A)}$ dynamical variables are all described in terms of the "fundamental"/true variables of the, say, crystal ions of which there are only $N^{(F)}$.

Approximate Description of Only $N^{(F)}$ True Variables as a Larger Number $N^{(A)}>>N^{(F)}$ of Formal Variables

We want to approximate statistically a system of $N^{(F)}$ harmonic oscillators with Hamiltonian

$$
\begin{aligned}
H^{(F)} & =\sum_{n}\left(\frac{1}{2} p_{n}^{(F) 2}+\frac{1}{2} \omega^{2} q_{n}^{(F) 2}\right) \\
{\left[q_{m}^{(F)}, p_{n}^{(F)}\right] } & =i \hbar^{(F)} \delta_{n m}
\end{aligned}
$$

by a system of $N^{(A)}>>N(F)$ harmonic oscillators, with variables $\left(q_{l}^{(A)}, p_{l}^{(A)}\right)$, that are in reality just functions - say linear functions - of the set of $N^{(F)}$ variables, as if this "formal system" of $N^{(A)}$ variables $q_{l}^{(A)}$ formed an indepent set of $N^{(A)}$ harmonic oscillators.

\section{Identifying a Couple of Sets of Harmonic Oscillators}

We want to approximate the "fundamental":

$$
\begin{aligned}
H^{(F)} & =\sum_{n}^{N^{(F)}}\left(\frac{1}{2} p_{n}^{(F) 2}+\frac{1}{2} \omega^{2} q_{n}^{(F) 2}\right) \\
{\left[q_{m}^{(F)}, p_{n}^{(F)}\right] } & =i \hbar^{(F)} \delta_{n m} \\
\text { by } & \text { the "formal approximation" } \\
H^{(A)} & =\sum_{l}^{N^{(A)}}\left(\frac{1}{2} p_{l}^{(A) 2}+\frac{1}{2} \omega^{2} q_{l}^{(A) 2}\right)
\end{aligned}
$$




$$
\left[q_{k}^{(A)}, p_{l}^{(A)}\right]=i \hbar^{(A)} \delta_{k l}
$$

where we have relations of the form

$$
\begin{aligned}
& q_{l}^{(A)}=\sum_{n}^{N^{(F)}} B_{l n}^{(q)} q_{n}^{(F)} \\
& p_{l}^{(A)}=\sum_{n}^{N^{(F)}} B_{l n}^{(p)} p_{n}^{(F)} .
\end{aligned}
$$

We have two systems of d.o.f. $\left(q_{n}^{(F)}, p_{n}^{(F)}\right)$ and $\left(q_{l}^{(A)}, p_{l}^{(A)}\right)$, which we want to treat as harmonic oscillators connected by relations of the form

$$
\begin{aligned}
& q_{l}^{(A)}=\sum_{n}^{N^{(F)}} B_{l n}^{(q)} q_{n}^{(F)} \\
& p_{l}^{(A)}=\sum_{n}^{N^{(F)}} B_{l n}^{(p)} p_{n}^{(F)}
\end{aligned}
$$

where the non-diagonal/rectangular matrices $B_{l n}^{(q)}$ and $B_{l n}^{(p)}$ are transition matrices between the two different systems of oscillators; they will be treated only statistically as being for our purpose random.

If we simply played the game, that our two sets of degrees of freedom each had only one single mass for all the d.o.f. in the set, so that

$$
\begin{aligned}
& p_{l}^{(A)}=m^{(A)} \dot{q}_{l}^{(A)} \\
& p_{l}^{(F)}=m^{(F)} \dot{q}_{l}^{(F)},
\end{aligned}
$$

we would obtain the relation

$$
B_{l n}^{(p)}=\frac{m^{(A)}}{m^{(F)}} B_{l n}^{(q)} ;
$$

but if we want the kinetic term form simply $\frac{1}{2} \sum_{l=1}^{N^{(A)}} p_{l}^{(A) 2}$ for the "formal"set of systems, say the matrix elements, and $\frac{1}{2} \sum_{n=1}^{N^{(F)}} p_{n}^{(F) 2}$ for the "fundamental" set, we must take $m^{(A)}=m^{(F)}=1$.

\section{Relations between the $\omega$-Parameters}

In order that the two systems of sets of harmonic oscillator be related by

$$
\begin{aligned}
& q_{l}^{(A)}=\sum_{n}^{N^{(F)}} B_{l n}^{(q)} q_{n}^{(F)} \\
& p_{l}^{(A)}=\sum_{n}^{N^{(F)}} B_{l n}^{(p)} p_{n}^{(F)}
\end{aligned}
$$

shall represent the same physics, we take that they at least have the same total kinetic and the same total potential energies.

Identifying Potential and Kinetic Energies for the Two Systems: 


$$
\begin{aligned}
\frac{1}{2} \sum_{l=1}^{N^{(A)}} p_{l}^{(A) 2} & =\frac{1}{2} \sum_{n=1}^{N^{(F)}} p_{n}^{(F) 2} \\
\frac{1}{2} \omega^{(A) 2} \sum_{l=1}^{N^{(A)}} q_{l}^{(A) 2} & =\frac{1}{2} \omega^{(F) 2} \sum_{n=1}^{N^{(F)}} q_{n}^{(F) 2}, \quad \text { which implies } \\
\sum_{l=1}^{N^{(A)}} B_{l n}^{(p)} B_{l m}^{(p)} & =\delta_{n m} \text { and } \\
\omega^{(A) 2} \sum_{l=1}^{N^{(A)}} B_{l n}^{(q)} B_{l m}^{(q)} & =\omega^{(f) 2} \delta_{n m} .
\end{aligned}
$$

We get a Normalized $B_{l n}$. Taken this we obtain especially that $\sum_{l=1}^{N^{(A)}} B_{l n}^{(p)} B_{l m}^{(p)}$ is zero for $n \neq m$, while it for all cases of $n=m$ (but fixed) has the same value, so that we can call it $B=$ $\sum_{l=1}^{N^{(A)}} B_{l n}^{(p)} B_{l n}^{(p)}$ (no summation over n). Actually we find from identical kinetic energies that $\mathrm{B}=1$.

Relations to Approximately Identify Two Systems with Different Numbers of Degrees of Freedom.

Using the definition $B=\sum_{l=1}^{N^{(A)}} B_{l n}^{(p)} B_{l n}^{(p)}$ (no summation over n) we get:

From same kinetic energies: $B=1$

From same potential energies: $\quad \omega^{(A) 2} B=\omega^{(F) 2}$

or simply the $\omega$ 's must be the same.

How are the Commutators Connected?

The commutators for a $q_{l}^{(a)}$ and a $p_{k}^{(A)}$ are evaluated as

$$
\begin{aligned}
& {\left[q_{l}^{(A)}, p_{k}^{(A)}\right]=\sum_{n, m=1}^{N^{(F)}} B_{l n}^{(q)} B_{k m}^{(p)} \approx} \\
& \approx \frac{B N^{(F)}}{N^{(A)}},
\end{aligned}
$$

which is very small if $N^{(F)}<<N^{(A)}$ !

$$
\hbar^{(A)}=\hbar^{(F)} * \frac{N^{(F)}}{N^{(A)}} .
$$

Effective Classical Approximation for Homolumo-gap-effect.

The result

$$
\hbar^{(A)}=\hbar^{(F)} * \frac{N^{(F)}}{N^{(A)}} .
$$

is very welcome/useful for justifying the calculational technology in our Homolumo gap paper with Andric, Jonke and two of us (DJ and HBN) [ㅍ] , because in order to come through the calculation we effectively had to use what corresponds to ignoring the quantum fluctuations and so using in reality a classical approximation. In this mentioned article we take all matrix elements in a somehow chopped off matrix single particle Hamiltonian for the electron/fermion as independent Harmonic oscillator variables. 


\section{Our Homolumo-gap Paper uses Too Many Variables, Thus goes to Classical}

But that is far too many variables in a genuine macroscopic piece of matter, which should have only a number of bosonic d.o.f. going up with increasing the size of the piece of matter only as proportional to the volume (to the first power, NOT to the second!)

According to the above we want to conclude, that the main change to repair for this physically bad correspondence of our model to a genuine macroscopic piece of material is that we shall use a negligible Planck constant for the too many dynamical matrix elements in our published model. But actually it happened that we finally approximated away this Planck constant anyway, and so the above rather became a justification for what we did earlier.

\section{Abstract Derivation of Homolumo-gap Formulation}

In this section (which is really a new idea that might be best for a separate paper if not pure nonsense ?) we would like in a very abstract formulation to stress how generally a "homolumo gap effect formulation", much like the one in the papers of two of us with Andric and Jonke, is to be understood. Really we want to make an abstract formulation with the interaction being represented by a (matrix-like) symbol with four indices $V_{a b}^{c d}$, the indices being in correspondence with a basis in the space of single particle electron states. For simplicity to avoid unimportant problems we shall even think of there being only a finite number of index values, although strictly speaking there would in real physics be infinitely many electron states.

Now the procedure and first approximation is that we seek the ground state of the many fermion (= electron) system of the form of a certain sub-hilbert space of the space of single particle electron states being filled, while the rest is empty. Such a state of the second quantized system is in fact characterized formally by just a projection operator P say on the single particle hilbert space. In our matrix like notation it would of course be a projection matrix $P_{a}^{b}$ with the properties of a projection matrix

$$
\begin{aligned}
P_{a}^{b} & =\left(P_{b}^{a}\right)^{*} ; \\
P_{a}^{b} P_{b}^{c} & =P_{a}^{c} .
\end{aligned}
$$

The average of the interaction given by $V_{a b}^{c d}$ is then

$$
E_{V}=P_{a}^{b} P_{c}^{d} V_{b d}^{a c}
$$

For simplicity we leave out the energy of the free particles, but it would be easy to add.

It is now the main spirit of the present section to define an effective state-dependent piece of the Hamiltonian for the single particle (electron)

$$
M_{a}^{b}=V_{a c}^{b d} P_{d}^{c}
$$

This definition of $M_{a}^{b}$ is at least so that is gives the energy $E_{V}$ when the state from $P_{a}^{b}$ is inserted.

Now we can ask what happens, if we take one electron out of the sea and put it into an unfilled state. Can that be done with an infinitesimally small extra energy, or is there some lower limit? That is the question of whether there is a homolumo gap. 
It should be already stressed, that even the very general ansatz for the effective single particle hamiltonian (8.4) can be considered an on the background depending dynamical piece of single particle hamiltonian, like the (difference) $M-M_{0}$ in the above notation, is a significant/non-trivial assumption. The effective linearized Hamiltonian $M_{a}^{b}$ from (8.4) obviously depends on the state given by $P_{a}^{b}$, which we shall think of as being adjusted to minimize the total energy.

If we define the state from which we move an electron by its projection operator $P_{\mathbf{h} a}^{b}$, where $\mathbf{h}$ stands for hole, and the state to which we move it by its projection $P_{\mathbf{p} a}^{b}$, we obtain the energy of the excitation by this movement as

$$
\begin{aligned}
\Delta E & =\left(P_{\mathbf{p} a}^{b}-P_{\mathbf{h} a}^{b}\right) M_{b}^{a} \\
& =\left(P_{\mathbf{p} a}^{b}-P_{\mathbf{h} a}^{b}\right) V_{b c}^{a d} P_{d}^{c} .
\end{aligned}
$$

From the minimization condition w.r.t. varying to the set of states filled $\Psi_{a k}$ where $k=1,2, \ldots$ enumerates the set of filled states (in an ansatz for seeking the ground state), so that the projection matrix is

$$
P_{a}^{b}=\sum_{k} \Psi_{a k} \Psi_{k}^{* b}
$$

we deduce as usual taking the derivatives at a minimum the derivative being zero condition:

$$
\begin{aligned}
& 0=\left(\sum_{k} \delta \Psi_{a k} * \Psi_{k}^{* b}\right) P_{c}^{d} V_{c d}^{a b} \\
& \text { or } \quad 0=\psi_{k}^{* b} P_{c}^{d} V_{c d}^{a b} \quad(\text { for all } k \text { ) } \\
& \text { or } \quad 0=\delta P_{a}^{b} P_{c}^{d} V_{b d}^{a c} \quad \text { (for variations } \delta P_{a}^{b} \text { respecting projection property of } P_{a}^{b} \text { ). }
\end{aligned}
$$

Now the general spirit of the homolumo gap effect would in this language translate into, that considering the homo-level, say for the index $k=h$, i.e. $\psi_{a h}$ for which $M_{a}^{b}$ has the highest filled eigenvalue

$$
\begin{array}{cl}
M_{a}^{b} \Psi_{b h}=\lambda_{h} \Psi_{a h} & \left(\text { where } \lambda_{h} \text { is highest filled eigenvalue }\right) \\
M_{a}^{b} \Psi_{b l}=\lambda_{l} \Psi_{a l} & \left(\text { where } \lambda_{l} \text { is lowest unfilled eigenvalue }\right)
\end{array}
$$

and varying it by $\delta \Psi_{a h}$ in the direction with a component in a empty level would lead to genuinely modify the second quantized state of the whole system and thus also $M_{a}^{b}$, and especially matrix elements between filled and empty levels. If now there were no homolumo-gap the variation of the matrix elements between homo and lumo would give linear variation, and thus the differentiability would be violated so that the derivative condition for minimization would not be fulfilled.

This at least should be true barring some special fine-tuning. But such special fine tuning would mean, it would seem at first, that there must be some symmetry.

Let us attempt a general argumentation like this:

Having a condition like ( $8 . \mathrm{C}$ ) means that one can change the filled states $\Psi_{a k}$ by small amounts $\delta \Psi_{a k}$ and obtain only second or higher order corrections to the total energy $E_{V}$ as represented in (8.3). However, if the homolumo-gap size is

$$
\Delta_{\text {homolumo }}=\lambda_{l}-\lambda_{h},
$$


then, if a certain variation $\delta \Psi_{a h}$ of the homo-level state by rotating it into the lumo-state $\Psi_{a l}$ can be considered "small" - of first order and is denoted "first o." one would effectively have the modified levels come out by diagonalizing a matrix like

$$
\text { "matrix" }=\left(\begin{array}{cc}
\lambda_{l}+\text { "first o." } & \text { "first o." } \\
\text { "first o." } & \lambda_{h}+\text { "first o." }
\end{array}\right) \text {. }
$$

Thus until second order terms become important one would get a lowering of the homo-level $\lambda_{h}$ compared to the lumo-one $\lambda_{l}$ provided the difference at first $\Delta_{\text {homolumo }}$ is much smaller than the first order terms.

We therefore conclude that it is not possible for the homolumo-gap $\Delta_{\text {homolumo }}$ to be much smaller than the second order term in the expansion of $M_{a}^{b}$ as a function of the variation $\delta \Psi_{a h}$ say.

If we thus have a general estimate of the second order term order of magnitudewise for such expansion, this would give us the expected order for the homolumo-gap $\Delta_{\text {homolumo }}$.

\section{Conclusion}

- Our "old" model, in which dark matter consists of cm-big pearls with masses of the order of 100 thousand ton to half a million ton, was suggested to be able to provide the "unidentified" $\mathrm{X}$-ray line of $3.5 \mathrm{keV}$ in fact by:

- Providing the line as a result of excitons (= an electron and a hole pair) annihilating, the homolumo-gap should then be essentially $3.5 \mathrm{keV}$.

- The distribution of the $3.5 \mathrm{keV}$ signal over the sky did not fit exactly the distribution of dark matter, but rather is more concentrated around the centres of big galaxies or galaxy clusters. This suggests that the $3.5 \mathrm{keV}$ radiation does not simply come equally strongly from all dark matter!

- For instance a production of the $3.5 \mathrm{keV}$ radiation in connection with collision (e.g. annihilation) of dark matter with itself would be more favoured as the source.

- Remarkably "our pearl model" produces, by the collision of the dark matter pearls, a huge amount of energy, of the order of one tenth ${ }^{1}$ of the Einstein energy of a pearl and this energy production gives the correct order of magnitude of the observed intensity of the $3.5 \mathrm{keV}$ line.

\subsection{Other Achievements of Our Dark Matter Model}

In addition to the well fitting of the $3.5 \mathrm{keV}$ line, we claim a good fitting, when we identify one of our pearls with the object that fell in Tunguska in 1908 and made the trees fall in a $70 \mathrm{~km}$ extended region and produced a huge "fire" on the sky....

- The rate of there falling one pearl on the earth about once every 100 or 200 years fits well with the surface tension being of the order given - by dimensional arguments - from the weak interaction scale $\sim 100 \mathrm{GeV}$.

\footnotetext{
${ }^{1}$ By the more detailed estimation in [5]] by two of us it seems that it is rather $1 / 100$.
} 
- And with it producing Kimberlite Pipes, of which one found $\sim 6500$ on the earth. (You find them on old cratons, where you have the oldest geological layers.)

\subsection{Successes of our Multiple Point Principle}

The Multiple Point Principle among other predictions says: Inside-pearl and OutsidePearl Phases have the Same Energy Density, if no matter present.

This new law of Nature - a fine tuning model - has its own successes:

- Two of us - CDF and HBN - PREdicted the Higgs mass to be $135 \mathrm{GeV} \pm 10 \mathrm{GeV}$ long before the Higgs was found experimentally.

- It "solves" the hierarchy problem in the sense that it says: Let us fine tune! Make a finetuning theory and then an exponentially low Higgs or weak scale comes out relative to say the Planck scale [四].

- We could get three independent estimates for the mass of the bound state of 6 top +6 anti top quarks, which we expect. We suggested that these bound states fill the vacuum inside the dark matter pearls. These estimates all turned out order of magnitudewise to be $\sim 750 \mathrm{GeV}$.

\subsection{On our Development of Calculating Homolumo-gap}

We have adjusted a bit the previous work by two of us and I. Andric and L. Jonke [1]], in order to make it a bit more appropriate for being applied to a genuine macroscopic system: Actually it is for such a system not so realistic to think of a matrix in the space of all electronic states - even if restricted to only a few bands - with all matrix elements being dynamical and independent degrees of freedom. The number of degrees of freedom for such a matrix would namely go proportionally to the square of the extensive quantities of the macroscopic system, rather than just proportionally to say the volume.

We find that, after curing that problem, an effective very small Planck constant for the dynamical matrix elements is achieved.

We also have put up a more abstract formalism aiming at seeing the homolumo gap as an extremely general phenomenon once one has interactions between the "fermions".

\section{References}

[1] C. D. Froggatt and H. B. Nielsen, Phys. Rev. Lett. 95 (2005) 231301 [arXiv:astro-ph/0508513]

[2] C.D. Froggatt and H.B. Nielsen, "Dark matter from encapsulated atoms," Bled Workshops Phys. 6 (2005) no.2, 32-39 Prepared for Conference: C05-07-19.3 Proceedings [arXiv:astro-ph/0512454]

[3] C. D. Froggatt and H. B. Nielsen, “Tunguska Dark Matter Ball,” Int. J. Mod. Phys. A 30 (2015) no.13, 1550066

[4] C.D. Froggatt and H.B. Nielsen, Trying to understand the Standard Model parameters. Invited talk by H.B. Nielsen at the "XXXI ITEP Winter School of Physics", Moscow, Russia, 18-26 February, 2003; Surveys High Energy Phys. 18, 55-75 (2003); hep-ph/0308144.

[5] C.D. Froggatt, H.B. Nielsen and L.V. Laperashvili, Int. J. Mod. Phys. A 20, 1268 (2005); hep-ph/0406110. 
[6] C.D. Froggatt and H.B. Nielsen, Phys. Rev. D 80, 034033 (2009); arXiv:0811.2089.

[7] C.D. Froggatt, H.B. Nielsen, Hierarchy Problem and a New Bound State, in Proc. to the Euroconference on Symmetries Beyond the Standard Model, p.73, Slovenia, Portoroz, 2003 (DMFA, Zaloznistvo, 2003); arXiv: hep-ph/0312218.

[8] C.D. Froggatt, The Hierarchy problem and an exotic bound state, in: Proceedings of 10th International Symposium on Particles, Strings and Cosmology,

(PASCOS 04), Boston, Massachusetts, 16-22 Aug, 2004. Published in: "Boston 2004, Particles, strings and cosmology", pp.325-334; hep-ph/0412337.

[9] C.D. Froggatt, L.V. Laperashvili and H.B. Nielsen, $A$ New bound state $6 t+6$ anti-t and the fundamental-weak scale hierarchy in the Standard Model, in: Proceedings of 13th International Seminar on High-Energy Physics: QUARKS-2004, Pushkinskie Gory, Russia, 24-30 May, 2004; hep-ph/0410243.

[10] C.D. Froggatt, L.V. Laperashvili and H.B. Nielsen, Phys. Atom. Nucl. 69, 67 (2006) [Yad. Fiz. 69, 3 (2006)]; hep-ph/0407102.

[11] C.D. Froggatt, L.V. Laperashvili, R.B. Nevzorov and H.B. Nielsen, The Production of $6 t+6 \bar{t}$ bound state at colliders. A talk given by H.B. Nielsen at CERN, 2008, preprint CERN-PH-TH/2008-051.

[12] C.D. Froggatt, L.V. Laperashvili, R.B. Nevzorov, H.B. Nielsen and C.R. Das, New Bound States of Top-anti-Top Quarks and T-balls Production at Colliders (Tevatron, LHC, etc.), Report CHEP-PKU-1-04-2008, CERN (2008); arXiv:0804.4506.

[13] C.D. Froggatt and H.B. Nielsen, ICHEP 2008, Philadelphia, Pennsylvania; arXiv:0810.0475.

[14] C.R. Das, C.D. Froggatt, L.V. Laperashvili and H.B. Nielsen, Int. J. Mod. Phys. A 26, 2503 (2011); arXiv:0812.0828.

[15] C.D. Froggatt, C.R. Das, L.V. Laperashvili and H.B. Nielsen, New indications of the existence of the 6 top-anti-top quark bound states in the LHC experiments, a talk by L.V. Laperashvili at the Conference "Quarkonium-2012", Moscow, Russia, MEPhI, November, 12 - 16, 2012, arXiv:1212.2168 [hep-ph]; Yad. Fiz., 76, 172 (2013).

[16] C.D. Froggatt, C.R. Das, L.V. Laperashvili, H.B. Nielsen, Int. J. Mod. Phys. A30, 1550132 (2015); arXiv:1501.00139.

[17] I. Andric, L. Jonke, D. Jurmanet and H.B. Nielsen, Int. J. Mod. Phys. A 32, 1750046 (2017).

[18] E. Bulbul, M. Markevitch, A. Foster et al., ApJ. 789, 13 (2014);

E. Bulbul, M. Markevitch, A. Foster et al., arXiv:1409.4143;

E. Bulbul, M. Markevitch, A. Foster et al., ApJ. 831, 55 (2016).

[19] A. Boyarsky, O. Ruchayskiy, D. Iakubovskyi and J. Franse, Phys. Rev. Lett. 113, 251301 (2014);

A. Boyarsky, J. Franse, D. Iakubovskyi and O. Ruchayskiy, Phys. Rev. Lett. 115, 161301 (2015).

[20] A. Merle and A. Schneider, Phys. Lett. B749, 283 (2015).

[21] T. Jeltema and S. Profumo, MNRAS, 450, 2143 (2015).

[22] K.J.H. Phillips, B. Sylwester and J. Sylwester, ApJ. 809, 50 (2015).

[23] Hitomi Collboration, Nature 535, 117 (2016);

Hitomi Collaboration, ApJ.837, L15 (2017).

[24] T. Jeltema and S. Profumo, MNRAS 458, 3592 (2016). 
[25] O. Ruchayskiy, A. Boyarsky, D. Iakubovskyi et al., MNRAS 460, 1390 (2016).

[26] D. A. Iakubovskyi Adv.Astron.Space Phys. 6, 1 (2016).

[27] E. Carlson, T. Jeltema and S. Profumo, JCAP 2, 009 (2015).

[28] M. C. Smith, G. R. Ruchti, A. Helmi et al., MNRAS. 379, 755 (2007).

[29] D.L. Bennett and H.B. Nielsen, Int. J. Mod. Phys. A9, 5155 (1994).

[30] D.L. Bennett, C.D. Froggatt and H.B. Nielsen, in Conference C94-07-20, Proc. 27th Int. Conf. on High Energy Physics, Glasgow, Scotland, 1994, eds. P. Bussey and I. Knowles (IOP Publishing Ltd., 1995), p. 557.

[31] D.L. Bennett, C.D. Froggatt and H.B. Nielsen, in Conference C94-09-13, Perspectives in Particle Physics ÂŠ 94 , eds. D. Klabu.car, I. Picek and D. Tadi.c (World Scientific, Singapore, 1995), p. 255, [hep-ph/9504294].

[32] C.D. Froggatt and H.B. Nielsen, Phys. Lett. B368, 96 (1996).

[33] H. B. Nielsen and C. D. Froggatt, "Influence from the future" Proceedings of the 5th Hellenic School and Workshops on Elementary Particle Physics; Corfu 1995, Conference C95-09-03.1, [arXiv:hep-ph/9607575].

[34] D. L. Bennett, PhD thesis at The Niels Bohr Institute, Copenhagen, "Multiple Point criticallity. Fine tuning and Fundamental Physics: Prediction for Gauge Couplings gives $\alpha^{-1}=136.8 \pm 9 . " 31$. May, 1996.

[35] D.L. Bennett and H.B. Nielsen, Int. J. Mod. Phys. A14, 3313 (1999).

[36] C.D. Froggatt, L.V. Laperashvili, R.B. Nevzorov and H.B. Nielsen, Phys. Atom. Nucl. 67, 582 (2004) [Yad. Fiz. 67, 601 (2004)], arXiv:hep-ph/0310127.

[37] C.D. Froggatt, L.V. Laperashvili and H.B. Nielsen, Phys. Atom. Nucl. 69, 67 (2006), hep-ph/0407102.

[38] D.L. Bennett, L.V. Laperashvili and H.B. Nielsen, Relation between fine structure constants at the Planck scale from multiple point principle, in Proc. 9th Workshop: What Comes Beyond the Standard Models, Bled, Slovenia, eds. M. Breskvar et al. (DMFA, Zaloznistvo, Ljubljana, 2006), p. 10, arXiv:hep-ph/0612250.

[39] D.L. Bennett, L.V. Laperashvili and H.B. Nielsen, Fine structure constants at the Planck scale from multiple point principle, in Proc. 10th Workshop on What Comes Beyond the Standard Model, Bled, Slovenia, 2007, arXiv:0711.4681.

[40] C.D. Froggatt, R.B. Nevzorov and H.B. Nielsen, Smallness of the cosmological constant and the multiple point principle, J. Phys. Conf. Ser. 110, 072012 (2008), arXiv:0708.2907.

[41] C.D. Froggatt, R.B. Nevzorov, H.B. Nielsen, A.W. Thomas, Phys. Lett. B737, 167 (2014), arXiv:1403.1001.

[42] C.D. Froggatt, R.B. Nevzorov, H.B. Nielsen, A.W. Thomas, On the smallness of the cosmological constant in SUGRA models with Planck scale SUSY breaking and degenerate vacua, 2015 European Physical Society Conference on High Energy Physics (EPS-HEP 2015), 22-29 Jul, 2015, Vienna, Austria; arXiv:1510.05379.

[43] Yuta Hamada, Hikaru Kawai, and Kiyoharu Kawana, Prog. Theor. Exp. Phys. (2015) 123B03.

[44] Hamada Y., Kawai H., and Oda K. Y., Phys. Rev. D 87, 053009 (2013); 89, 059901 (2014). 
[45] Kiyoharu Kawana, Prog. Theor. Exp. Phys. (2015) 023B04

[46] Yuta Hamada, Hikaru Kawai, and Kiyoharu Kawana, Int. J. Mod. Phys. A 29, 1450099 (2014)

[47] C. D. Froggatt, The Top quark and other fermion masses, Proceedings of the 5th Hellenic School and Workshops on Elementary Particle Physics; Corfu 1995, Conference C95-09-03.1, [arXiv:hep-ph/9603432].

[48] C. D. Froggatt, The Problem of quark and lepton masses, Conference: C95-08-24.1, p.12-19 Proceedings of the Seventh Lomonosov Conference on Elementary Particle Physics, Moscow 1995, [hep-ph/9511400].

[49] C.D. Froggatt and H. B. Nielsen, Dynamical determination of the top quark and Higgs masses in the standard model, Contributed to Conference: C96-07-25, ICHEP96, Warsaw 1996, [hep-ph/9607302].

[50] C. D. Froggatt, H. B. Nielsen and D. J. Smith, Dynamical determination of the top quark and Higgs masses and fermion masses in the antigrand unification model Presented at Conference: C96-07-25, Proceedings of ICHEP96, Warsaw 1996, p.1127-1130, [hep-ph/9707205].

[51] C.D. Froggatt, Origin of mass, Surveys High Energy Phys. 18, 77 (2003); [hep-ph/0307138].

[52] C. D. Froggatt, H. B. Nielsen and Y. Takanishi, "Standard model Higgs boson mass from borderline metastability of the vacuum,” Phys. Rev. D 64 (2001) 113014; [hep-ph/0104161].

[53] H. B. Nielsen, D. L. Bennett, C. R. Das, C. D. Froggatt and L. V. Laperashvili, PoS CORFU2016 050 (2017); arXiv:1705.10749.

[54] H. B. Nielsen and C. D. Froggatt, Conference: C15-09-01, PoS CORFU2015 (2016) 021.

[55] M. Paszkowski and J. W. Mietelski, Acta Physica Polonica B44, 787 (2013)

[56] C. D. Froggatt and H. B. Nielsen, Mod. Phys. Lett.A30, no.36, 1550195 (2015).

[57] C. D. Froggatt and H. B. Nielsen, "The $3.5 \mathrm{kev}$ line from non- perturbative Standard Model dark matter balls", article soon to appear. 